\title{
ABHANDLUNGEN・ARTICLES・ARTICLES
}

\section{Rechtstransfer nach Afrika?}

\author{
Christian Roschmann ${ }^{1}$
}

\begin{abstract}
In a globalized international system of economic competition, development cannot take place without adequate legal structures which have to be adopted from industrialized countries. Their adoption, however, meets with three kinds of obstacles: lack of processing capacities, cultural habits and power structures. To overcome those and institutionalize a state of the law including human rights, several structural changes in African societies and states have to be made simultaneously (as they condition each other), the empowerment of civil society, the creation of mass incomes, the changing of notions of legitimacy and the transfer of legal structures. This calls for a two-pronged approach, engineering those changes in a coordinated fashion on the one hand and the direct transfer and implementation of specific laws in new and hitherto unregulated fields on the other.
\end{abstract}

\section{Problemlage}

Die Beschäftigung mit Rechtstransfer, die in der Vergangenheit von einem überwiegend akademischen Interesse bestimmt war, ${ }^{2}$ wird seit einiger Zeit zunehmend unter anderen Blickwinkeln vorgenommen, vor allem bezüglich ihrer Praxisrelevanz für die Entwicklungspolitik, und daneben teilweise für die Verbreitung eines europäisch-amerikanischen Menschen- und Staatsverständnisses, das in vielen Bezügen gleichzeitig als entwicklungskausal gesehen wird. Die Leitbegriffe sind Rule of Law und Menschenrechte.

Entsprechendes Recht wird als notwendiger Kausalfaktor bei der Industrialisierung von Entwicklungsländern gesehen, entwickeltes Recht als unentbehrlich ${ }^{3}$ für die Strukturierung

1 Christian Roschmann is Professor at Harz University of Applied Sciences in Halberstadt, Germany, and was previously head of the "Konrad-Adenauer-Stiftung Rule of Law Programme for AfricaSubsahara" (E-mail: croschmann@hs-harz.de).

2 Literaturnachweise etwa bei Horst Dutt, Entwicklungszusammenarbeit durch Rechtsberatung, Berlin 1994, S. 19; Oliver Meinecke, Rechtsprojekte in der Entwicklungszusammenarbeit. Theorie und Praxis am Beispiel von GTZ-Projekten zur Konsolidierung des Rechtsstaats in Südafrika und Sambia, Schriftenreihe der Hochschule Speyer Band 188, Berlin 2007, S. 55 ff.

$3 \mathrm{Zu}$ den Perzeptionen individueller oder kollektiver Akteure im Einzelnen, s. etwa Cornelia Glinz, Verwaltungsrecht und Rechtsstaatlichkeit in Namibia. Ein entwicklungspolitisches Reformprojekt, Baden-Baden 2013, S. 119. Schönberger spricht von einer "Gefällelage" zwischen bisherigem und zu transferierendem Recht bezüglich der Erreichung vorteilhafter kollektiver Positionen, Christoph 
des Entwicklungsweges wie auch darüber hinaus in manchen Bezügen als Agent of Change in einer Anschubfunktion.

Die hierfür notwendigen rechtlichen Strukturen sollen - durch Rechtstransfer - den Rechtssystemen der industrialisierten Länder entnommen werden.

Während in den 1950er Jahren die Law-and-Development Bewegung, ${ }^{4}$ deren Theoriehintergrund einen Teilbereich der auf der US-amerikanischen behavioristischen Wissenschaftstradition fußenden politikwissenschaftlichen Modernisierungstheorien darstellte, ${ }^{5}$ versucht hatte, Rechtsstrukturen, insbesondere rationale Verwaltungsstrukturen, ${ }^{6}$ von den Industriestaaten, insbesondere den USA, instrumentell ${ }^{7} \mathrm{zu}$ übertragen, versuchen nach deren Scheitern Ansätze, deren Fokusse sich unter den Begriffen Good Governance und Rule of Law zusammenfassen lassen, in einer eher holistischen und inkrementalistischen Vorgehensweise die Interdependenz von Recht und anderen Faktoren für die Entwicklungszusammenarbeit zu thematisieren und beschäftigen sich auch mit der Untersuchung von Gesetzmäßigkeiten.

So besteht heute weitgehend Einigkeit darüber, dass ohne eine entsprechende rechtliche Strukturierung

- sich eine Volkswirtschaft nicht entwickeln kann, hierzu bedarf es unter anderem der Möglichkeiten, Kapitalgesellschaften zu gründen, der Existenz eines Kreditsicherungs-

Schönberger, Verwaltungsrechtsvergleichung: Eigenheiten, Methoden und Geschichte, in: Armin von Bogdandy, Sabino Cassese, Peter. M. Huber (Hrsg.), unter Mitwirkung von Diana Zacharias, Handbuch Ius Publicum, Band IV, Verwaltungsrecht in Europa: Wissenschaft. Heidelberg 2011, S. 493-549, S. 515.

4 Hierzu Brun-Otto Bryde, Die Erfahrungen der Law and Development Diskussion und die Transformationsforschung, in: Michael Kirk, Jost Kramer, Rolf Steding (Hrsg.): Die Rolle des Rechts im Entwicklungsprozess, Referate der Fachgruppe „Grundlagenforschung“ anlässlich der Tagung für Rechtsvergleichung im September 1985, Frankfurt a.M. 1986, S. 9-36 Nachweise auch bei Jacqueline Neumann, Die Förderung der Rule of Law in der Entwicklungszusammenarbeit. Ein Beitrag zur Herausbildung einer völkerrechtlichen Verfassungsnorm. Berlin 2013, S. 276; vgl. auch Meinecke, Fn 2, S. 118.

5 Lucian Pye/Sidney Verba, Political Culture and Political Development, Princeton N. J., 1965. David E. Apter, Some Conceptual Approaches to the Study of Modernization, Englewood Cliffs N. J., 1968.

6 Joseph La Palombara (ed), Bureaucracy and Political Development, Princeton N. J., 1963; Fred Riggs, Administration in Developing Countries. The Theory of Prismatic Society, Boston Ma., 1964. Deutschsprachige Übersichten bei Roman Schnur, Über vergleichende Verwaltungswissenschaft, Verwaltungsarchiv 52 (1961), S. 1- 24; Alfred Diamant/Hans Jecht, Verwaltung und Entwicklung. Forschungstendenzen und Modelle in den USA, Die öffentliche Verwaltung 19 (1966), S. 378-393; Hans-Georg Steiffert, Forschungsansätze zu "Verwaltung und politische Entwicklung", in: Dieter Oberndörfer (Hrsg.), Verwaltung und Politik in der Dritten Welt, Berlin 1971, S. 237-299.

7 Riggs, Fn 5; Fred Riggs, Relearning an Old Lesson: The Political Context of Development Administration, Public Administration Review 26 (1965), S. 70-79, weitere Nachweise bei Dutt, Fn 2, S. 42. 
und eines Insolvenzrechts und nicht zuletzt einer gesicherten Eigentumsordnung, wobei Rechte insbesondere auch durchsetzbar sein müssen.

- eine soziale Entwicklung nicht möglich ist; hierzu sind u. a. Subsistenzsicherung, Arbeitsschutzbestimmungen, Kranken- und Rentenversicherungen unerlässlich.

- bei steigendem Wirtschaftswachstum die Umwelt nicht geschützt werden kann gegen Raubbau, Verseuchung und, in neuerer Zeit, insbesondere auch gegen Klimaveränderungen und deren Folgen, ${ }^{8}$ die auch erheblich entwicklungsrelevant sind, ${ }^{9}$ und zu deren Schutz auch Entwicklungsländer bei den internationalen Bemühungen zur Eindämmung von Ursachen und Linderung von Folgen notwendig mitwirken müssen.

Auch wenn man von der Problematik der global commons wie dem Klima absieht, ist Abkopplung vom internationalen konkurrenzkapitalistischen System (im Sinne von Verweigerung von Kommunikations- oder zumindest Waren- und Dienstleistungsaustauschen mit anderen Staaten) und damit dem freiwilligen Verzicht auf Entwicklung heute für kein Land eine denkbare Alternative. ${ }^{10}$ In allen Entwicklungsländern besteht vielmehr ein starkes Bedürfnis nach Entwicklung. Sie haben auf panafrikanischer Ebene die Anerkennung eines Rechts auf Entwicklung festgeschrieben ${ }^{11}$ und auf UN-Ebene die Anerkennung eines solchen Rechts durchgesetzt. ${ }^{12}$

Daher befinden sich Entwicklungsländer in der historisch neuen Dilemmasituation globaler Einbindung, in der sie wirtschaftlicher Entwicklung nicht ausweichen können, selbst wenn sie dies wollten, da sie sonst ökonomisch zurückblieben und verarmten, wobei Entwicklung nur in rechtlichen Strukturen möglich ist, über die sie jedoch großenteils nicht verfügen.

\section{Bisherige Lösungsansätze}

Es liegt nun nahe, den Blick auf diejenigen Länder zu werfen, die die angestrebte Entwicklung bereits erfolgreich durchgemacht haben und neben den volkswirtschaftlichen und so-

8 Oliver C. Ruppel. Christian Roschmann, Katharina Ruppel-Schlichting (eds), Climate Change: International Law, Global Governance. Volume 1. Legal Responses and Global Responsibility, Baden-Baden 2013.

9 Oliver C. Ruppel, Intersections of Law and Cooperative Global Climate Governance - Challenges in the Anthropocene, in: Oliver C. Ruppel, Christian Roschmann, Katharina Ruppel-Schlichting (eds): Climate Change: International Law Global Governance. Volume I. Legal Responses and Global Responsibility, Baden-Baden 2013, S. 29-94.

10 Kaum jemand würde etwa dazu zu bewegen sein, auf Fernseher und Handy zu verzichten, wohl am wenigsten in Entwicklungsländern, wo solche auch Statussymbole darstellen.

11 Art. 22 African Charter on Human's and People's Rights von 1981.

12 Declaration on the Right to Development der UN-Vollversammlung 1986, A/RES/41/128. Auch wenn dies kein in einem Abkommen festgeschriebenes Recht darstellt, wurde es in Art. 10 der Vienna World Conference on Human Rights (1993) und in Principle 3 der Rio Declaration on Environment and Development (1992) bestätigt, vgl. hierzu etwa Christian Tomuschat, Human Rights. Between Idealism and Realism, Oxford 2003, S. 48. 
zialen Mechanismen auch deren entsprechende Rechtsstrukturen zu übernehmen, wie das erfolgreiche Nachholer wie Japan, Korea oder auch die Türkei zu verschiedenen Zeiten schon mehrfach vorgemacht haben. Hinzu kommt, dass in Afrika die institutionellen Grundlagen, wie auch diverse mehr oder weniger ausgeformte Rechtsstrukturen, meist von Industrieländern ${ }^{13}$ als Kolonialmächten bereits ohne Beteiligung der Akteure vor Ort dort eingeführt wurden. ${ }^{14}$

Im akademischen Diskurs beschäftigt sich eine breite Diskussion mit der Frage, ob und gegebenenfalls unter welchen Bedingungen, der Transfer von Recht von einem Staat in den andern und insbesondere von einer Kultur in die andere erfolgreich möglich ist ${ }^{15}$. Hierbei stehen sich im Wesentlichen zwei Lager gegenüber. Die Vertreter des einen meinen, Rechtsinstitute seien ohne größere inhaltliche Verluste transferierbar, ${ }^{16}$ die anderen ${ }^{17}$ sind der gegenteiligen Auffassung und halten dies für im Wesentlichen nicht möglich.

Die erstere Ansicht glaubt, eine Reihe, auch historischer Beispiele für sich anführen zu können. So nennt Watson die erfolgreiche Übernahme römisch-rechtlicher Rechtsfiguren in Kontinentaleuropa und in Schottland, ebenso wie Beispiele aus der Antike. Für die Gegenwart könnte auf die äthiopische Übernahme des französischen Zivilgesetzbuches verwiesen werden. Eine sehr weitgehende Variante ist die Vorstellung, Recht gerade in der Form spezifischer Rechtsnormen sei Ausdruck einer ahistorischen kosmischen Ordnung, ${ }^{18}$ die immer, überall und notwendig gleich sei. Hierunter fällt heute vor allem die vorherrschende Meinung islamischer Rechtsschulen. ${ }^{19}$

13 Mit Ausnahme von Spanien und Portugal.

14 Brun-Otto Bryde, The Politics and Sociology of African Legal Development, Frankfurt a. M. 1976; Harald Sippel, Afrikanisches Gewohnheitsrecht zwischen indigener Handlungskompetenz und europäischen Einflüssen, in: Harald Fischer-Tiné, (Hrsg.), Handeln und Verhandeln. Kolonialismus, Transkulturelle Prozesse und Handlungskompetenz, Münster/ Hamburg/London 2002, S. 197-223.

15 Hierzu etwa David Nelken, Towards a Sociology of Legal Adaptation, in: David Nelken, Johannes Feest (eds) Adapting Legal Cultures, Portland Or 2001, S. 7-54 mwN, der allerdings (S. 8) den Begriff "legal transplants" als Angriff auf "legal sociology" und unvereinbar mit "internal evolution" sieht.

16 Alan Watson, Legal Transplants. An Approach to Comparative Law, Edinburgh 1974; William Ewald, Comparative Justice II, The Logic of Legal Transplants, American Journal of Comparative Law 43 (1995), S. 489-510.

17 Pierre Legrand, What "Legal Transplants"?, in: Nelken, David, Feest, Johannes (eds), Adapting Legal Cultures, Portland Or 2001, S. 55-69; Lawrence Friedman, The Concept of Legal Culture. A Reply, in: Nelken, David (ed) Comparing Legal Cultures, Aldershot 1997, S. 33-40; Roger Cotterell, Is there a Logic of Legal Transplants? in: Nelken, David/ Feest, Johannes (eds), Adapting Legal Cultures, Portland Or 2001, S. 71-91; Otto Kahn-Freund, On Uses and Misuses of Comparative Law, in: Modern Law Review 37 (1974), S. 1-27; Robert B. Seidman, The State, Law and Development, London 1978.

18 Michel Alliot, Über die Arten des „Rechts-Transfers“, in: Wolfgang Fikentscher, Herbert Franke und Oskar Köhler (Hrsg.), Entstehung und Wandel rechtlicher Traditionen, Freiburg/München 1980, S. 161-234, S. 173.

19 Hierzu Neumann, Fn 4, S. 278. 
Die Vertreter der Gegenansicht ${ }^{20}$ weisen darauf hin, dieselben Rechtsstrukturen hätten in verschiedenen kulturellen Umwelten niemals dieselbe Bedeutung, da sie erst in kulturellen Kontexten und durch diese ihre Interpretation erführen. Im Einzelnen werden eine Reihe von unterschiedlichen Faktoren in unterschiedlichen Situationen für perzipierte Erfolge oder Misserfolge ${ }^{21}$ von "transplants“ verantwortlich gemacht. ${ }^{22}$ Je unterschiedlicher der kulturelle Kontext ${ }^{23}$ und, damit verwoben, der wirtschaftliche und politische sei, desto unterschiedlicher seien die inhaltlichen Bedürfnisse nach Regulierung im Einzelnen sowie Ziele, Funktionsmechanismen und Wirkungen von Rechtsfiguren. ${ }^{24}$ Die Einnahme der Gegenposition mache die notwendige Untersuchung kultureller Vorverständnisse und damit eine hermeneutische Beschäftigung mit Rechtsstrukturen unmöglich. ${ }^{25}$ Schließlich entspreche die Trennung von Rechts- und Sozialordnung in Entwicklungsländern nicht der von Industrieländern, ${ }^{26}$ weil in Entwicklungsländern der Zusammenhang dieser Ordnungen meist ein anderer sei, ${ }^{27}$ was sowohl in Werten wie Zielen und Mitteln zum Ausdruck komme.

Eine Variante dieses Theoriestrangs meint, Recht sei nur innerhalb des Denksystems einer Kultur schaffbar, was nicht nur Transferierbarkeit sondern auch Rechtsvergleiche unmöglich machen würde. ${ }^{28}$ Es sei daher fraglich, ob es überhaupt möglich ist, europäische Rechtsvorstellungen auf Entwicklungsländer zu übertragen. ${ }^{29}$

Bei näherer Betrachtung erscheinen die offenbar antagonistischen ${ }^{30}$ Positionen jedoch akademisch überhöht und konvergieren letztlich. Auch die Befürworter einer Übertragbarkeit von Recht sehen die kontextuellen Veränderungen, die Rechtsnormen in verschiedenen staatlichen, aber auch zeitlichen, Handlungszusammenhängen erfahren ${ }^{31}$ und führen Beispiele an, wie solche in zeitlicher und räumlicher Distanz, teils bis zur Unkenntlichkeit, ver-

20 Etwa Seidman, Fn 17; Legrand, Fn 17; Friedman 1997, Fn 17; Lawrence Friedman, A History of American Law, 2nd edition, New York 1985.

21 Nelken weist auf die unterschiedliche Erfolgskarriere derselben Transplants in Russland und Polen hin, Nelken, Fn 15, S. 35.

22 Nelken, Fn 15, S. 41.

23 Die "Kluft zwischen den Systemen", Meinecke, Fn 2, S. 134 mwN hierzu.

24 Seidman, Fn 17, S. 34: "legal transplants... practically never work".

25 Legrand, Fn 17, S. 58.

26 Dutt, Fn 2, S. 37.

27 Wolfgang Fikentscher, Herbert Frank, Oskar Köhler, Über Aufgaben und Möglichkeiten einer historischen Rechtsanthropologie, in: Wolfgang Fikentscher, Herbert Frank, Oskar Köhler (Hrsg.), Entstehung und Wandel rechtlicher Traditionen, Freiburg/München 1980, S. 15-52, S. 37 führen Beispiele an, wie diese Ordnungen in umfassende kosmische Ordnungen integriert seien.

28 Wolfgang Fikentscher, Synepeik und eine synepeische Definition des Rechts, in: Wolfgang Fikentscher, Herbert Franke, Oskar Köhler (Hrsg.): Entstehung und Wandel rechtlicher Traditionen, Freiburg/München 1980, S. 53-120, S. 97.

29 S. Dutt, Fn 2, S. 38, Meinecke, Fn 2, S. 116-118.

30 Hierzu Cotterell, Fn 17, S. 74 und auch zu ihrer (jedenfalls teilweise als möglich gesehenen) Konvergenz.

31 Glinz, Fn 3, S. 124 mnN. 
ändert wurden. Recht wird in dieser Sichtweise allmählich dekonstruiert und, von einem dogmatischen Standpunkt aus, ,verfälscht“. ${ }^{32}$ Auf der anderen Seite gibt es erfolgreiche Übernahmen von Gesetzen wie das türkische Zivilgesetzbuch von der Schweiz, das äthiopische von Frankreich und das japanische von Deutschland. Watson stellt fest: ,most changes in most systems are the result of borrowing”, 33 fügt aber dann hinzu: ,, reception almost always involves a change in the law". ${ }^{34}$ Hier wird klar: der Fokus dieser Schule liegt auf der Karriere juristischer Institutionen. ${ }^{35}$

Auf der anderen Seite geben die Anhänger der These kontextueller Bedingtheiten des Rechts $\mathrm{zu}$, dass unter bestimmten Bedingungen und mit bestimmten Modifikationen Rechtskonstruktionen von einem staatlichen Handlungszusammenhang in einen andern übernommen werden können und konzedieren, die Einzelheiten würden entscheiden. ${ }^{36}$ Der Blickwinkel dieser Schule richtet sich auf die Bedingtheiten von Rechtskonstruktionen. ${ }^{37}$ Rechtsnormen werden umgebildet und angepasst an neue Verhältnisse. ${ }^{38}$ Für diesen Prozess werden rechtssoziologische Ansätze als erkenntnisleitend identifiziert. ${ }^{39}$ Als entscheidend wird die Einbettung rechtlicher Normen in einen sozialen und kulturellen Rahmen gesehen. ${ }^{40}$ Hier kann sich dann die Frage stellen, ob „Recht“" in der Form des kulturellen, vor allem semantischen, „Packings“" wie es in Europa entwickelt wurde, für Afrika nutzbar gemacht werden kann ${ }^{41}$ oder eher als Hindernis empfunden wird. ${ }^{42}$

Es handelt sich letztlich bei diesem Meinungsstreit nicht um eine Kontroverse über die faktische Möglichkeit der erfolgreichen Transferierbarkeit von Recht, sondern um den Vorrang von Blickwinkeln, dem rechtsdogmatischen oder dem rechtssoziologischen. Beide Ansätze gehen mehr oder weniger gleichermaßen von einer Transferierbarkeit bei vergleichbaren Bedingungen und Problemlagen aus, wenn der Nutzen des Transfers die Kosten übersteigt, und halten gleichzeitig Adaptionsleistungen im Detail für notwendig. Beide Ansätze sind jedoch für die entwicklungspolitische Praxis nicht zielführend. Sie erklären beide nicht, welche spezifischen Bedürfnislagen, Bedingtheiten und Hindernisse für erfolgreiche Rechtstransfers gerade in Entwicklungsländern und insbesondere in Afrika bestehen.

32 Alliot, Fn 18, S. 212; Alliot zeigt auch Beispiele und Techniken der "Zerstörung" von übertragenem Recht auf (S. 211).

33 Watson, Fn 16, S. 95.

34 Watson, Fn 16, S. 97.

35 Und sie "missversteht" wohl nicht die Rolle der Rechtssoziologie, wie Cotterell (Fn 17, S. 77) meint.

36 Nelken, Fn 15, S. 21 ff.

37 Sie betrachtet Faktoren wie die „Ähnlichkeit betreffender Gesellschaften”(Alliot, Fn 18, S. 173).

38 Alliot, Fn 18, S. 212.

39 Nelken, Fn 15, passim.

40 Nelken, Fn 15, S. 25.

41 Fikentscher, Fn 28, S. 99.

42 Fikentscher, Fn 28, S. 87: Letztlich bedürfe es einer Metatheorie des Denkens, um diese Phänomene adäquat zu erfassen. 
Einen Schritt zu dieser Erklärung kann man in der Argumentation Teubners ${ }^{43}$ sehen. Teubner argumentiert differenzierend und mit systemtheoretischem Ansatz, Recht sei nicht mehr einheitlich mit anderen gesellschaftlichen Systemen gekoppelt. Seine Ansicht, Recht sei je nach Thematik mehr oder weniger locker und jeweils mit fragmentierten gesellschaftlichen Segmenten wie auch mit divergierenden ökonomischen Bedingungen verbunden, dürfte wohl auf Industrieländer im allgemeinen zutreffen, wie auch seine Beobachtung, dass Rechtstransplantate im juristischen wie in den damit gekoppelten anderen gesellschaftlichen Bereichen je unterschiedliche Adaptionen hervorrufen, die sich - wegen ihrer Koppelung - gegenseitig potentiell ,irritieren“.

Diese Überlegungen kann man differenzierend wohl auch auf Entwicklungsländer übertragen. Man hat es dort allerdings mit einem kleinen, in Modernisierung betroffenen Bereich zu tun, insbesondere bei der zu entwickelnden Wirtschaft, wo ökonomische, aber meist nicht soziale und politische Strukturen mit neuen rechtlichen verbunden sind, während der umfangreiche traditionelle Bereich von beiden weitgehend abgekoppelt und für Transplantate unerreichbar ist, so dass „Irritationen“ teils stärker, teils schwächer auftreten als in Industrieländern.

Übertragenes Recht wird in Entwicklungsländern in vielen Lebensbereichen als Fremdkörper angesehen und fordert in großem Maßstab institutionelle ${ }^{44}$ wie psychische Adaptionsleistungen. Wir haben es daher hier mit der Frage kritischer Massen zu tun. Wieviel Adaption kann institutionell und psychisch bewältigt werden? Während es sich etwa bei der von Teubner beschriebenen Problematik der Übernahme der römisch-rechtlichen bona fides Regel ins angelsächsische Recht ${ }^{45}$ um eine begrenzte Problematik handelt, stellt die Übernahme ganzer Bereiche westlicher Rechtssysteme und ihrer Systemlogik ungleich umfangreichere und tiefergehende Adaptionsanforderungen an afrikanische Gesellschaften und Individuen, wird mit entsprechenden rechtlichen Strukturen ja auch ein großer Teil der Lebenswelt verändert. Und der Erfolg einer Rechtsübertragung kann, wie Cotterell zu Recht bemerkt, ${ }^{46}$ nur danach bemessen werden, ob sie die beabsichtigten Wirkungen erzielt. Hier stellt sich dann aber auch die Frage, ob Geber und Nehmer dieselben Wirkungen erzielen wollen. Denn auch Fassaden und die Nicht-Erreichung von Zielen können Ziele sein, wie

43 Gunther Teubner, Rechtsirritationen. Zur Koevolution von Rechtsnormen und Produktionsregimes, in: Günter Dux, Frank Welz (Hrsg.), Moral und Recht im Diskurs der Moderne. Zur Legitimation gesellschaftlicher Ordnungen, Opladen 2001, S. 351-380, der allerdings Industrieländer, besonders Großbritannien, im Blick hat.

44 Im weitesten Sinne, insbesondere auch Handlungspfade umfassende.

45 Teubner, Fn 43, S. 370.

46 Cotterell, Fn 17, S. 79. 
Seibel in seinem 1991 erschienenen Beitrag „Erfolgreich Scheiternde Organisationen“47 überzeugend darstellt.

Problematisch ist daher die Aufgabe der Zusammenführung von Lebens- (einschließlich Vorstellungs- und Werte-) Welt und entwicklungsnotwendiger Rechtsstruktur. ${ }^{48} \mathrm{Je}$ weiter diese auseinander klaffen, desto schwieriger und unwahrscheinlicher wird eine erfolgreiche Adaptation von Rechtstransplantaten. Recht wird im Extremfall von Beteiligten als Mittel zur Zerstörung gesellschaftlicher Strukturen gesehen. ${ }^{49}$ Entsprechend wird auch ein Mangel an lokaler „ownership“ bei Reformbemühungen durch unkritischen Transfer von Organisationen der Entwicklungszusammenarbeit festgestellt. ${ }^{50}$ In der Regel bestehe ein formeller, noch nicht legitimierter Kanon neben einem hergebrachten informellen, legitimierten. ${ }^{51}$ So weist Neumann etwa auf die soziale Gruppenzugehörigkeit als informeller aber im Wesentlichen einzig ergebnisbestimmender Faktor bei Streitschlichtungen, die offiziell nach formalen Regeln erfolgen, hin. ${ }^{52}$ Dies kann im schlimmsten Fall zu einem „Rechtsvakuum“53 führen in dem Sinne, dass alte Handlungsstrukturen sich auflösen und neue sich noch nicht gebildet haben, verstärkt durch ein Legitimitätsvakuum bezüglich des neuen Rechts, ${ }^{54}$ das nur Fassadenfunktion entfalten kann.

Die Chance für eine erfolgreiche Rechtsadaption sind je höher, desto mehr gemeinsame Anknüpfungspunkte Gesellschaften in ihrer Lebenswelt haben, vor allem ein vergleichbares Welt- einschließlich Selbstbild mit ähnlichen Lebensbedingungen und ähnlichen sozialen Erscheinungsformen ${ }^{55}$ und damit ähnlichen Gerechtigkeitsvorstellungen. Dies kann sichtbar gemacht werden an der Unvereinbarkeit von konfrontativen, auf kontradiktorischen Positionen beruhenden, forensischen verwaltungsrechtlichen Kontrollinstrumenten mit ostasiatischen auf der Herstellung von Harmonie als Wert beruhenden Mechanismen im Umgang von Bürger und Staat, ${ }^{56}$ die zur Ablehnung westlicher Rechtskultur führen kann.

Alliot beobachtet, eine Rechtsübertragung gelinge bei einer „Ähnlichkeit im Selbstverständnis der beiden Gesellschaften“, es erfolge dann eine „Auflösung des transferierten

47 Wolfgang Seibel, Erfolgreich scheiternde Organisationen. Zur politischen Ökonomie des Organisationsversagens, in: Politische Vierteljahresschrift 32 (1991) S. 479-496; s. auch Alex Jettinghoff, State Formation and Legal Change: On the Impact of International Politics, in: Wolfgang Fikentscher, Herbert Franke und Oskar Köhler (Hrsg.), Entstehung und Wandel rechtlicher Traditionen, Freiburg/München 1980, S. 99-116, S. 106 zu Darstellungseffekten in der internationalen Arena.

48 Dutt, Fn 2, S. 40.

49 Alliot, Fn 18, S. 215.

50 Neumann, Fn 4, S. 280.

51 Neumann, Fn 4, S. 282.

52 Neumann, Fn 4, S. 282.

53 Dutt, Fn 2, S. 45.

54 Dutt, Fn 2, S. 47.

55 Alliot, Fn 18, S. 173; Schönberger, Fn 3, S. 543.

56 Eun-Jeun Lee, Der soziale Rechtsstaat als Alternative zur autoritären Herrschaft. Zur Aktualisierung der Staats- und Demokratietheorie Hermann Hellers, Berlin 1994, S. 131. 
Rechts selbst“" ${ }^{57}$ Recht, so wird postuliert, müsse an veränderte Umstände angepasst werden. ${ }^{58}$ Rezipiert werden könne nur ,ad modum recipientis“ .59

\section{Hindernisse erfolgreichen Rechtstransfers}

Die Praxis der Entwicklungszusammenarbeit in Afrika findet jedoch meist eine diesem Leitbild in keiner Weise entsprechende typische Situation vor: Nämlich die Existenz von andersartigen, sich überlagernden und konkurrierenden Rechtsordnungen ${ }^{60}$ und -vorstellungen verschiedener Bevölkerungsgruppen, die lediglich durch koloniale Ordnungen in einen Staat zusammengefasst wurden, manchmal auch innerhalb derselben Bevölkerungsgruppe und vor allem innerhalb eines Ordnungsrahmens der „Recht“ in unserem Sinn und andere Ordnungsvorstellungen nicht trennt. ${ }^{61}$

Daneben tritt dann die Situation der Innovation völlig neuer Rechtsgebiete, die erst im Zug wirtschaftlicher Entwicklung entstehen. Gerade diese aber fordern geförderte Entwicklung und damit die Entwicklung neuer Handlungsformen und Tätigkeitsfelder, besonders im Staats- und im Wirtschaftsrecht, die wiederum der Innovation rechtlicher Strukturierungen bedürfen, die dann der Strukturierung entsprechender Handlungsfelder in Industrieländern entnommen werden. ${ }^{62}$ Die Lebens- und Rechtswelt in Afrika entspricht also in der Regel gerade nicht den Voraussetzungen der Ähnlichkeit.

Da bestimmte Rechtsinstrumente jedoch offenbar als Vehikel für nachholende Entwicklung benötigt werden und solche durch die Industrieländer bereitgestellt werden, soll nun der Frage nachgegangen werden, welche der spezifischen, sich aus der Verschiedenheit der Lebensverhältnisse ergebenden Hindernisse, als die identifiziert werden können, die einer Übernahme und entsprechender bedarfsgerechter Modifizierung - oder auch der Bildung entsprechender autochthoner analoger Rechtsinstrumente - entgegenstehen. Hier sind im Wesentlichen drei zu nennen:

57 Alliot, Fn 18, S. 210.

58 Schönberger (Fn 3, S. 518) spricht von „Rekonfiguration“, Wiater (Patricia Wiater, Kulturpluralismus als Herausforderung für Rechtstheorie und Rechtspraxis. Eine völkerrechtsdogmatische und ethnologische Auseinandersetzung mit der Rechtssprechung des EGMR. Leipziger Schriften zum Völkerrecht, Europarecht und ausländischem öffentlichen Recht, Band 15, Baden-Baden 2009, S. 103) von „Rechtsakkulturation“.

59 Schönberger, Fn 3, S. 519.

60 Fikentscher, Fn 28, S. 70; Neumann, Fn 4, S. 281.

61 Dutt, Fn 2, S. 38.

62 Dutt, Fn 2, S. 44. 


\section{Institutionelle Verarbeitungskapazität}

Das Erste ist die institutionelle Verarbeitungskapazität. ${ }^{63}$ Hierunter ist nicht nur die Prozesskapazität zu verstehen, wie etwa die Fähigkeit eines Parlaments, einen Gesetzgebungsprozess qualitativ ${ }^{64}$ erfolgreich von Anfang bis Ende durchzuführen, oder einer Behörde, in geordneten Verfahren die Ergebnisse dieses Prozesses zu implementieren ${ }^{65}$, sondern auch und vor allem - die institutionelle Kohärenz des Staatsapparates. Hierunter ist das regelhaft abgestimmte Zusammenwirken der verschiedenen Staatsorgane zu verstehen. Staatsorgane müssen ineinandergreifend und auf einander abgestimmt agieren können. Ineinandergreifende Institutionen können nicht in ihrer Organisationstiefe heterogen strukturiert sein, ohne dass es zu suboptimalen Handlungsergebnissen kommt. Staatsorgane dürfen des Weiteren nicht in die Kompetenzbereiche anderer Staatsorgane eingreifen, sei es aus Unkenntnis oder bewusster Ausnutzung von Organisationsmängeln. Staatsorgane müssen die regelhaft zustande gekommenen Entscheidungen anderer Staatsorgane sowohl respektieren, als auch sie zur Grundlage ihres eigenen Handelns machen können. Der Abstimmungsprozess muss sinnvoll strukturiert und kenntnisvoll durchgeführt werden können. Ein typisches Beispiel fehlenden Ineinandergreifens ist die Missachtung von Gesetzen und richterlichen Entscheidungen durch die Exekutive; ${ }^{66}$ ein solches für mangelnde institutionelle Kohärenz aufgrund unterschiedlicher Organisationstiefe ist die fehlende Möglichkeit, Grundsteuern einzuziehen, wenn das Katasterwesen einen wesentlich niedereren Organisationsgrad aufweist, als das Steuerwesen.

Ebenfalls unter den Begriff mangelnder institutioneller Verarbeitungskapazität (in Form von Organisations- und Supervisionsmängeln) gefasst werden kann das Problem fehlender Professionalität staatlicher Funktionsträger. Hierbei handelt es sich sicher um ein Problem mangelhafter Ausbildung und in der Folge Kompetenz, aber auch um eines der Berufsethik. Die Koordination und Beachtung von Regeln muss weiter von der Öffentlichkeit überwacht werden können und erzwingbar sein. Sonst stößt auch die Herausbildung einer entsprechenden Berufsethik auf große Hindernisse.

Des Weiteren stellen Kommunikationsprobleme ein faktisches Hindernis dar. Sehr viele Individuen erreicht die Nachricht rechtlicher Neuerungen sehr spät oder gar nicht, so dass diese nicht in einzuübenden Handlungszusammenhängen Gemeingut werden können.

In der Verkennung der faktischen Verarbeitungskapazität lag ein Grund für das Scheitern der Law and Development-Bewegung. Es reicht eben nicht aus, westliche Regeln als Gesetze in die Welt zu setzen, wenn diese nicht beachtet werden, teils weil sie unbekanntes

63 Hierzu Meinecke, Fn 2, S. 61, der insbesondere auf die erhebliche Gefahr, dass ein EZ-gefördertes Gesetz nicht umgesetzt werden könne, hinweist.

64 Meinecke, Fn 2, S. 95.

65 Meinecke, Fn 2, S. 97 mwN, S. 101 ff.

66 Vgl. zu dieser Problematik Oliver C. Ruppel, The Role of the Executive in Safeguarding the Independence of the Judiciary in Namibia, in: Horn, Nico, Bösl, Anton (eds): The Independence of the Judiciary in Namibia, Windhoek 2008, S. 207-228. 
Gedankengut darstellen, der Umgang mit ihnen nicht eingeübt ist, ihnen eine Legitimitätsbasis fehlt und kein Beamter sicher ist, ob er nicht der Einzige wäre der das Gesetz beachtete und sich dadurch außerdem die Feindschaft aller anderen Beteiligten zuzöge, teils aber auch, weil sie nicht beachtet werden können. So sind nach detaillierten Regeln rechtsfehlerfrei zustande gekommene strafrechtliche Verurteilungen bedeutungslos, wenn ein Mechanismus zu ihrer Vollstreckung fehlt. Die Problematisierung fehlender institutioneller Verarbeitungskapazität betrifft hauptsächlich das Können, nicht das Wollen. Dass beides zusammenhängt, soll im Folgenden aufgezeigt werden.

\section{Kultur}

Das zweite Hindernis kann mit dem allgemeinen und sehr unspezifischen ${ }^{67}$ Begriff „,Kultur“ bezeichnet werden. Der Blickwinkel unter dem „Kultur“ betrachtet wird, ist nicht der des Nicht-Könnens sondern der des Nicht-Wollens der Übernahme rechtlicher Strukturen (auch wenn er sich bezüglich des Themas der Berufsethik mit jenem überschneidet). Die Übernahme von Normen wird abgelehnt, weil sie der inneren Welt der betroffenen Individuen widerspricht. Hierbei sind verschiedene Bereiche zu unterscheiden:

- Der Wahrnehmungsbereich. Hierzu gehört, dass es sich bei den übernommenen um Regeln eines meist unbekannten strukturellen Typs handelt, nämlich um Regeln mit einem wesentlich höheren Abstraktionsgrad und anderer Rationalität als den den Individuen bekannten, ${ }^{68}$ deren Befolgung nicht immer in einem klaren Wenn-Dann Schema internalisiert werden kann, und dass Verhaltensbereiche reguliert werden, die bisher nicht oder wesentlich anders reguliert waren und somit Handlungsunsicherheit hervorgerufen wird, die in der Regel durch den Rückgriff auf bekannte Verhaltensmuster und Ignorierung der neuen kompensiert wird.

- Pfadabhängigkeiten. Menschliches Handeln und Denken vollzieht sich im Wesentlichen pfadabhängig, in Routinen und Gewohnheiten, die eine entlastende Funktion haben, weil sie nicht mehr reflektiert zu werden brauchen. Eine Abkehr von gewohnheitsgetragenen Strukturierungen des sozialen Lebens, zu denen auch die Beachtung von Recht gehört, ist kostenträchtig ${ }^{69}$ und wird zu vermeiden versucht. Neuerungen werden auch deshalb so weit es geht ignoriert. Das gilt oft auch für Neuerungen, die Verbesserungen der Lebensverhältnisse darstellen oder nach sich ziehen, weil die Abweichung von Gewohnheiten des Denkens und Handelns meist als psychisch und manchmal auch materiell kostenträchtiger gesehen wird als die Beibehaltung der alten. Hinzu kommt, dass Recht

67 Cotterell, Fn 17, S. 73.

68 Die übernommenen Rechtsregeln sind meist von moralischen Geboten entkoppelt; der Umgang hiermit bedarf eines Differenzierungsniveaus, das vielerorts unbekannt ist.

69 In einem weiteren Wortsinn als dem der neoklassischen Ökonomie: Jede Art von Nachteilen, nicht nur finanziellen sondern etwa auch psychische Kosten umfassenden. Vgl. hierzu Christian Roschmann, Recht, Gerechtigkeit und ökonomisches Handlungsmodell, Baden-Baden 2003, S. 47 ff. 
besonders in Entwicklungsländern eng mit außerrechtlichem sozialem Rollenverhalten verwoben ist. ${ }^{70}$

- Ängste. Ängste sind mehr als das Unbehagen am Verlassen einer Gewohnheit. Ängste beziehen sich auf befürchtete darüberhinausgehende Schäden. In besonderen Fällen wird befürchtet, die eigene Gesellschaft könne sich ganz oder partiell auflösen durch neue Handlungspraktiken und Denkweisen oder das Verlassen alter, und besonders durch Beeinflussung der Wertestrukturen. Ein extremer Fall sind Angst vor Magie und psychische (oder genauer: psychopathologische) Zwänge, ${ }^{71}$ etwa der Rache der Götter bei Verlassen der Pfade der hergebrachten und von diesen als heilig angesehenen Ordnungen. In vielen archaischen Rechtsordnungen, auch alteuropäischen, werden deshalb manche bereits geringfügige Übertretungen mit dem Tode bestraft oder haben zumindest gravierende Rechtsnachteile zur Folge wie den Verlust des Rechtsstreits im frührömischen Legisaktionenprozess bei Versprechern beim Aufsagen der Klagformel.

- Legitimität. Recht stellt eine Werteordnung dar, die existiert und befolgt wird, weil sie auf Legitimität beruht, ${ }^{72}$ Gerechtigkeitsüberzeugungen entspricht. Jede Gesellschaft hat Werte verinnerlicht, an denen sie Handeln und Zustände misst. Diese Werte verkörpern die Legitimitätsstruktur an der auch Recht gemessen wird. ${ }^{73}$ Je statischer Gesellschaften sind, desto mehr hat Hergebrachtes die Vermutung der Richtigkeit und wird ihm ein kosmischer Bezug zugeschrieben. ${ }^{74}$ Es schafft per se Legitimität. Problematisch ist dies in Gesellschaften, in denen Unveränderlichkeit als solche kulturessentialistisch gesehen wird und auch einen inhaltlichen Wert und Teil der Legitimitätsstruktur darstellt wie in den meisten islamischen ${ }^{75}$ und die strukturelle mit inhaltlichen Komponenten so verschränken, ${ }^{76}$ dass Änderungen per se und ungeachtet ihres Inhalts als Unwert gesehen werden.

- Wenn transferiertes Recht zu verinnerlichten Werten in Widerspruch steht, erhält es keine Legitimität und wird abgelehnt und nicht befolgt. Hier hat auch das Problem fehlender Berufsethik ${ }^{77}$ eine seiner Wurzeln. Ein Auswechseln der Legitimitätsbasis, wie man sie bei aufgeklärten afrikanischen Mittelklassen beobachten kann, findet nur dann statt, wenn diese Auswechslung sich in einer Kosten - Nutzen Bilanz positiv auswirkt und

70 Dutt, Fn 2, S. 46.

71 Alliot, Fn 18, S. 186.

72 Max Weber, Wirtschaft und Gesellschaft, Tübingen 1972, S. 122 ff.

73 Weber, Fn 72, S. 122, Umfangreiche Nachweise zu dieser Problematik in entwicklungsspezifischer Sicht bei Meinecke, Fn 2, S. 98, Fn. 132.

74 Weber, Fn 72, S. 130 ff, Fikentscher, Franke, Köhler, Fn 27, S. 37.

75 Hierzu Alliot, Fn 18, S. 173.

76 Als kosmische Ordnungen, hierzu Alliot, Fn 18, S. 173.

77 S.o. Punkt 1 in diesem Kapitel. 
nicht psychisch zu kostenträchtig ist, d. h. nicht an die psychische Machbarkeitsgrenze stößt. ${ }^{78}$

- Gesellschaftliche Fragmentierung. Problematisch für die Akzeptanz transferierten Rechts ist die Fragmentierung von Gesellschaften in Afrika. Diese Länder sind zum guten Teil Schöpfungen der Kolonialmächte und die in ihnen lebenden Völker und Stämme haben oft verschiedene, wenn nicht antagonistische inhaltliche Vorstellungen von Legitimität oder Rechtsinhalten, ${ }^{79}$ zum Teil auch weil sie politisch in einem antagonistischen Verhältnis zueinander stehen. So ist es nicht ganz selten, dass einem Gesetzesvorhaben der einen Gruppe Widerstand von einer andern entgegengebracht wird, sei es aufgrund verschiedener Legitimitätsvorstellungen, sei es aus politischem Kalkül.

\section{Machtstrukturen}

Das dritte - und in der Praxis stärkste - Transferhindernis sind Machtstrukturen. Die politischen Strukturen in Entwicklungsländern korrelieren, wie in fast allen Ländern, mit den wirtschaftlichen. Es gibt keine Masseneinkommen und kaum eine Mittelklasse, eben infolge fehlender industrieller Entwicklung. Die große Masse der Bevölkerung bezieht ihr Einkommen aus der Landwirtschaft. ${ }^{80}$ Der Dienstleistungssektor ist, mit wenigen Ausnahmen, rudimentär ausgeprägt. Die Produktivität und das Prokopfeinkommen sind (außer in den Ölrentierländern) niedrig. Die höchsten Einkommen werden von Politikern und mit diesen klientelistisch verbundenen Gefolgsleuten (,Staatsklassen“) durch bürokratische Renten erzielt. ${ }^{81}$ An zweiter Stelle stehen solche aus Latifundien. Sehr erschwerend macht sich die Tatsache bemerkbar, dass diese Einkommen nur zu einem kleinen Teil in die eigene Volkswirtschaft zurückfließen. Industrialisierung würde dies ebenso grundlegend verändern wie sie das in den Industrieländern getan hat, die vor der Industrialisierung dieselbe Struktur aufwiesen. ${ }^{82}$

Politische und ökonomische Macht liegen bei Politikern und hochrangigen Bürokraten, die schnell Reichtum akkumulieren und auch Zugänge zu Erwerbschancen monopolisieren, insbesondere auch zu den wenigen lukrativen Marktpositionen wie etwa das Eigentum an Hotels mit internationalem Standard. Diese Strukturen liegen in der Unabhängigkeit des

78 Da Legitimitätsüberzeugungen in Weltbilder integriert sind, die kosmische Erklärungs- und Ordnungsvorstellungen darstellen, ist ihre Änderung eine tief in die individuellen Persönlichkeiten eingreifende. Vgl. Roschmann, Fn 69, S. 125 ff.

79 Fikentscher, Fn 27, S. 70.

80 Neben Tourismus und Bodenschätzen in einigen Ländern.

81 Hartmut Elsenhans, Abhängiger Kapitalismus oder bürokratische Entwicklungsgesellschaft. Versuch über den Staat in der Dritten Welt, Frankfurt a. M., 1981.

82 Dieter Senghaas, Von Europa lernen. Entwicklungsgeschichtliche Betrachtungen, Frankfurt a.M., 1982, wobei bei diesen allerdings die bürokratischen Renten viel niedriger waren, da es keine Geber gab, die diese gespeist hätten. 
Staatssektors ${ }^{83}$ infolge dessen Ausmaßes faktischer Unkontrollierbarkeit durch die Zivilgesellschaft begründet. Im Staatssektor entsprechend Positionierte können „Politics of Power" ${ }^{\text {84 }}$ betreiben. Macht liegt nicht bei abstrakten, „unpersönlichen“, regelgeleiteten Institutionen, sondern ist hoch personalisiert und ihre Ausübung damit extrem wandelbar und unberechenbar. Schwache Institutionalisierung korreliert daher mit Korruption. ${ }^{85}$ Bürokraten können im klientelistisch organisierten Staatsapparat ${ }^{86}$ nur durch persönliche Allianzen und Loyalitäten ,überleben“. ${ }^{87}$ Wie in allen vorindustriellen Gesellschaften ist die Öffentlichkeit infolge ihrer Armut machtlos und unorganisiert und infolge ihrer Machtlosigkeit unorganisierbar. Zivilgesellschaft gibt es deshalb nicht in nennenswertem Umfang. Sie kann insbesondere keine wirksame Kontrollfunktion wahrnehmen.

Wie in allen vorindustriellen Ländern umfasst unkontrollierbare und daher korruptive Machtausübung auch in Afrika die Anmaßung von Befugnissen, vom Staatspräsidenten bis zum Dorfpolizisten. Diese Strukturen werden gestützt durch ihre Einbettung in kulturelle Pfadabhängigkeiten wie auch Legitimitätsvorstellungen. ${ }^{88}$ Wenn in Afrika Herrscher seit unvordenklichen Zeiten unumschränkt, und in der Wahrnehmung der relevanten Öffentlichkeit zu Recht, über Leben und Tod geboten, stört sich das Volksempfinden nicht daran, wenn ein Staatspräsident sich entgegen der Verfassung ein x-tes Mal wählen lässt, wird diese ja ohnehin als ungemäßer, unverständlicher und bedeutungsloser Fremdkörper empfunden, oder wenn ein Polizist Schmiergelder verlangt, entspricht doch auch dies jahrtausendealter Praxis.

Wie die Eliten aller vorindustriellen Gesellschaften, ${ }^{89}$ haben auch die Eliten von Entwicklungsländern ein lebhaftes Interesse daran, dass sich der gegebene Zustand politischer und ökonomischer Machtverteilung nicht ändert. Sie machen meist große Anstrengungen, eine Änderung dieser Zustände zu verhindern. Denn Entwicklung schafft durch Massenmärkte Massenkonsum und Masseneinkommen und damit auf Kosten der Eliten ökonomisches und politisches empowerment breiterer Schichten, die dadurch eine Kontrollfunktion

83 Allgemein hierzu: Gabriel Almond, The Return of the State, in: American Political Science Review 36 (1988), S. 853-874; Theda Skocpol, Bringing the State Back in, Strategies of Analysis in Current Research, in: Peter. B. Evans, Dietrich Rueschemeyer, Theda Skocpol (eds): Bringing the State Back in, Cambridge Ma., 1985, S. 3-43; für Entwicklungsländer grundlegend: Elsenhans, Fn 81, s. auch Jettinghoff, Fn 47, S. 100.

84 Kahn-Freund, Fn 17, S. 23.

85 Dutt, Fn 2, S. 110, Christian Roschmann, Kann man den Begriff „Korruption” bei Verwaltungen der Dritten Welt anwenden?, in: Journal für Entwicklungspolitik 9 (1993), S. 417-426; Christian Roschmann, Bürokratie. zwischen Produktionsauftrag und Machtlogik, Baden-Baden 1999, S. 250.

86 Zur Beschreibung der Grundstruktur von klientelistisch organisierten Staatsapparaten, Elsenhans, Fn 81, S. $161 \mathrm{ff}$.

87 Und dies leider auch nicht ganz selten nicht nur im übertragenen Sinne.

88 Hierzu Roschmann 1993, Fn 85.

89 Insbesondere auch der europäischen, wie der Jahrhunderte währende Kampf des markt- und wettbewerbsorientierten Bürgertums mit rentenbeziehenden Kronen und Adligen zeigt. Vgl. hierzu Roschmann 1999, Fn 85, S. 103-182. 
wahrnehmen können und dies in der Regel auch tun. Starke Mittelklassen setzen Regeln und deren Einhaltung durch. Daher haben Eliten in Entwicklungsländern in der Regel ein vitales Interesse an der Verhinderung von Entwicklung und hintertreiben diese auf vielfältige Weise.

Ihren entsprechenden Aktivitäten wirken allerdings vier Faktoren entgegen.

- Es gibt immer unter Eliten entwicklungsorientiert gesinnte Individuen, die der Entwicklung ihres Landes Priorität vor Eigennutz geben.

- Es gibt unter Eliten immer Individuen oder Gruppen, die nicht sehen, dass die wirtschaftliche mit einer sozialen und politischen Dynamik einhergeht beziehungsweise die sich darüber keine Gedanken machen oder die glauben, sie könnten diese Dynamik beherrschen und in ihrem Sinne lenken. ${ }^{90}$

- Auch Eliten sind an Steigerung der Produktivität ihrer Volkswirtschaft interessiert, da sie durch Besetzung der lukrativeren Marktpositionen, meist unter Umgehung des Wettbewerbsmechanismus, an dieser überdurchschnittlich partizipieren. So besteht in den meisten Entwicklungsländern eine unklare Gemengelage von allgemeiner Entwicklungserschwerung und mehr situativer, marktfokussierter Entwicklungsförderung, jedenfalls solange hierdurch keine Änderung der Machtstrukturen erkennbar wird.

- Ein (nach Land unterschiedlich) großer Teil des Nationalprodukts geht auf Transferleistungen von Gebern zurück, die diese Leistungen von vorzeigbaren Entwicklungsbemühungen abhängig machen können und manchmal (in unterschiedlichem Maße) auch machen. Manipuliert wird diese Konditionalität allerdings durch den Aufbau einer Fassade scheinbar erfolgreicher Entwicklungsbemühungen, die jedoch so arrangiert werden, dass sie nicht durchschlagend zum Ziel führen können. ${ }^{91}$ Hierzu gehört auch die Verabschiedung von Gesetzen, von denen erwartet werden kann, dass sie nicht beachtet werden (können). Letztlich kommt es jedoch nicht darauf an, ob Gesetze nicht vorhanden sind oder nicht angewandt werden.

So stehen wir, wenn wir diese gegebenen Hindernisse betrachten, einerseits vor dem Befund schwer zu überwindend hoher Hindernisschwellen oder, anders gesagt, möglicherweise prohibitiv hoher Kosten, die den Zug der Entwicklung bremsen. Andererseits erfährt dieser Zug von hinten einen gewaltigen Druck durch die Zwänge der globalisierten Wirtschaft in Richtung Entwicklung. Diesem Dilemma kann sich kein Entwicklungsland entziehen. Es zwingt Entwicklungsländer, die Hemmnisse auszuräumen um nicht relativ zu verarmen und mögliche Entwicklung spät oder gar nicht durchzumachen

90 S. unten Fn 141.

91 Sie sind ,erfolgreich scheiternd” (Seibel, Fn 47). Diese Fassadentechnik wird weltweit angewandt und ist dann besonders erfolgreich, wenn sie in einem kollusiven (allerdings nicht immer als solchem reflektierten) Zusammenwirken angewandt wird, wobei auf der einen Seite die Eliten der Entwicklungsländer stehen und auf der andern die Sachwalter der Geber vor Ort, die Interesse am Erhalt ihrer meist überdurchschnittlich dotierten Positionen haben, sowie die politischen und administrativen Akteure in den Geberländern, die in ihrer nationalen Arena politische Erfolge vorzeigen wollen. 


\section{Umgang mit den Hindernissen}

Angesichts dieser Notwendigkeit ist es trotz aller theoretischen und praktischen Bemühungen nicht gelungen, die für wirtschaftliche, politische und gesellschaftliche Entwicklung notwendig gehaltenen rechtlichen Strukturierungen in die meisten Entwicklungsländer zu transferieren, insbesondere nach Afrika. Dies liegt an der ungenügenden Berücksichtigung der drei aufgezeigten Hinderniskomplexe, des institutionellen, das politischen und des kulturellen. Die Möglichkeit erfolgreichen Rechtstransfers steht und fällt mit dem adäquaten Umgang mit diesen Hindernissen.

Wie aber wurde nun mit diesen Hindernissen umgegangen und wie erfolgsführend sind die bisherigen Ansätze gewesen?

In den Anfängen der Entwicklungszusammenarbeit in den fünfziger und sechziger Jahren, entstand auf dem Theoriegebäude der politikwissenschaftlichen Modernisierungstheorien die Law and Development-Bewegung. ${ }^{92}$ Modernisierungstheoretische Ansätze gehen davon aus, Unterentwicklung sei das Ergebnis des Fehlens entsprechender politischer Institutionen. ${ }^{93}$ Solche müssten im Wesentlichen lediglich eingeführt werden, ${ }^{94}$ um Beachtung $\mathrm{zu}$ finden und dadurch Entwicklung in Gang zu bringen. Die Vertreter der Law-and-Development Bewegung wandten diese Betrachtungsweise auf Rechtsstrukturen allgemein, insbesondere auf die rechtliche Strukturierung politischer Institutionen, an und glaubten durch eine Verbindlicherklärung etwa von Verfassungen würden diese dann Wirkung entfalten. ${ }^{95}$

Die Vertreter modernisierungstheoretischer Ansätze mussten jedoch bald erkennen, dass die Verkündung von Verfassungen oder anderen Gesetzen noch nicht per se Wirkung entfaltet, sondern dass rechtliche Regelungsmechanismen Teile komplexer gesamtgesellschaftlicher Wirkungszusammenhänge sind. Gesetze, die am „grünen Tisch“ gemacht wurden, verschwanden in Archiven ${ }^{96}$ oder Vorschläge und Entwürfe wurden einfach ignoriert. Exemplarisch ist das gescheitere Großprojekt einer umfassenden Modernisierung des Iran durch Schah Reza Pahlawi, ${ }^{97}$ das in einer traditionalistischen Revolution und bewussten Abkehr von allen westlichen Werten endete.

92 S. Nachweise in Fn 4.

93 S. Riggs 1964, Fn 6; Riggs 1965, Fn 7; Apter, Fn 5; Gabriel Almond, Sidney Verba, The Civic Culture, Political Attitudes and Democracy in Five Nations, Princeton NJ 1963; La Polombara, Fn 6.

94 Riggs (1965, Fn 7, S. 77) etwa fordert, das „fundamental political framework“ müsse geändert werden. Es scheint, dass die Überzeugung, insbesondere bei US amerikanischen Wissenschaftlern, bestand, westliche Institutionen entwickelten eine Überzeugungskraft ihrer Überlegenheit aus sich heraus.

95 Diese Ansätze werden zu Unrecht auf Max Weber zurückgeführt, der eine rationale Herrschaft als die effizienteste Form staatlicher Herrschaft und als für Industrieländer notwendig ansah. Weber ging allerdings nicht davon aus, rationale Herrschaft sei voraussetzungslos. Er thematisierte sie auch nicht als Voraussetzung und schon gar nicht als beliebig schaffbare (s. Weber 1972, Fn 72 , S. $122 \mathrm{ff})$.

96 Dutt, Fn 2, S. 136.

97 Hierzu Dutt, Fn 2, S. 115. 
In der entwicklungstheoretischen Diskussion wurde dies nach und nach reflektiert und es etablierten sich zunächst Deutungsvarianten und Lösungsmodelle, die unter dem Begriff der Dependenztheorien bekannt wurden ${ }^{98}$ und in ihrer Mehrzahl marxistisch ausgerichtet waren. Sie führten Unterentwicklung auf Abhängigkeiten der Entwicklungsländer von den Industrienationen in globalen Handlungszusammenhängen zurück und forderten, ein Ende dieser Abhängigkeiten um Entwicklung in Gang zu bringen. Diese Ansätze erwiesen sich jedoch, auch als für die Frage von Rechtstransfers, nicht erkenntnisleitend. Ja, insgesamt erwies sich keiner der theoretischen Entwicklungsansätze als zielführend, so dass Menzel bereits 1992 vom „Scheitern der großen Theorie“" ${ }^{“ 99}$ sprach.

Seitdem bedienen sich die Bemühungen der Entwicklungszusammenarbeit in der Praxis pragmatischer, sowohl holistischer wie auch inkrementalistischer Ansätze, ${ }^{100}$ meist ohne expliziten Rückgriff auf akademische Theoriegebäude, wenn man vom Einfluss neoliberaler Ökonomen auf die Bretton Woods Institutionen in den Neunzigerjahren und etwas darüber hinaus absieht. Für die Frage des Rechtstransfers wurde von Praktikern der Begriff „Rule of Law“101 fruchtbar gemacht und von der Weltbank ${ }^{102}$ der der „Good Governance ${ }^{\text {“103 }}$ geprägt. ${ }^{104}$ Im Zentrum dieser Ansätze ${ }^{105}$ steht die Frage nach der Begrenzung staatlicher Macht. ${ }^{106}$ Sie implizieren eine Vorgehensweise, bei der die Interdependenzen ${ }^{107}$ von Recht und gesellschaftlichen Faktoren, etwa Akzeptanzen, oder faktischen Gegebenheiten, wie Zugang zur Justiz, ebenso berücksichtigt werden sollen wie mit wirtschaftlichen und insbesondere der Zusammenhang von Recht und dem politischen Sys-

98 Etwa Fernando H. Cardoso/Enzo Faletto, Abhängigkeit und Entwicklung in Lateinamerika, Frankfurt a. M. 1976.

99 Ulrich Menzel, Das Ende der Dritten Welt und das Scheitern der großen Theorie, Frankfurt a. M. 1992.

100 Meinecke, Fn 2, S. 53, 56.

101 Neumann, Fn 4, S. 205 zur Geschichte des Begriffs und der durch ihn ausgedrückten Sichtweise; s. auch Meinecke, Fn 2, S. 79, 82; grundsätzlich zu Rule-of-Law und Rechtsstaatlichkeit: Pietro Costa, The Rule of Law. A Historical Introduction, in: Pietro Costa, Danolo Zolo, (eds) with the Cooperation of Emilio Santoro, The Rule of Law, History, Theory and Criticism. Law and Philosophy Library Vol. 80, Dordrecht 2007, S. 73-152; Luc Heuschling, Etat de Droit, Rechtsstaat, Rule of Law, Paris 2002; Katharina Sobota, Das Prinzip Rechtsstaat, Tübingen 1997.

102 World Bank, Governance and Development, 1992; hierzu Graham Harrison, The World Bank and Africa. The Construction of Governance States, London 2004; Christian Theobald, Zur Ökonomik des Staates. Good Governance und die Perzeption der Weltbank, Baden-Baden 2001.

103 Zum Begriff Neumann, Fn 4, S. 220; Meinecke, Fn 2, S. 73; Tomuschat, Fn 12, S. 55 nennt als Komponenten: accountable government, civilsociety, professional bureaucracy.

104 Zum Verhältnis der beiden Begriffe vgl. Neumann, Fn 4, S. 292 ff.

105 Maik Falke, Die Deutsche Internationale Rechtsstaatsförderung zwischen Globalisierung und Nachhaltigkeit, in: Recht und Politik 46 (2010), S. 55-70, S. 63-67 sieht in ihrer Verfolgung einen "Systemwettbewerb".

106 Neumann, Fn 4, S. 211.

107 Falke, Fn 105, S. 61. 
tem. ${ }^{108}$ Eine Übertragung eigenen Rechts „eins zu eins“ wird abgelehnt und „Offenheit gegenüber anderen Rechtssystemen“ ${ }^{\text {"109 }}$ zum Prinzip erhoben. ${ }^{110}$

Im Zentrum der Bemühungen um Rechtstransfer stehen der staatliche Bereich, die Organisation des Staatsapparates und insbesondere das Verhältnis des Bürgers zum Staat, die zu Recht als wesentlich für Entwicklung angesehen werden. Es dürfte keine Frage sein, dass ein korrupter, indifferenter und leistungsschwacher Staatsapparat nicht die für eine geordnete Entwicklung notwendigen öffentlichen Güter produzieren kann.

An dieser Stelle ergeben sich jedoch zwei Fragen, die für erfolgreichen Rechtstransfer in Entwicklungsländer grundsätzlich sind:

1. Soll Recht die Straße sein, auf der sich Entwicklung bewegt, sozusagen, um ein Bild von Max Weber aufzugreifen, die Geleise, ${ }^{111}$ oder der Antrieb der Entwicklung, die Lokomotive?

2. Nach welchen Prinzipien muss ein Staatsapparat strukturiert sein, und zwar organisatorisch wie in seinem rechtlichen Verhältnis zum Bürger, um im Einzelfall entwicklungsförderlich zu sein und die beschriebenen Hindernisse auszuräumen? Für die Transferproblematik heißt das konkret: Welche Rechtsinstitute sollen transferiert werden und in welcher Gestalt?

Für die Beantwortung der ersten Frage hat das Scheitern der Law and Development-Bewegung deutlich gemacht, dass ein isolierter Transfer von Recht (einschließlich nach abstrakten Regeln konstituierter Institutionen), der nicht Ausdruck eines politisch-ökonomisch-gesellschaftlichen Willens und diesem entsprechender Arrangements ist, wie eine abgekoppelte Lokomotive im leeren Raum steht. Eine Adaption im Sinne einer Integration in einen Handlungszusammenhang mit den andern gesellschaftlichen Bereichen findet dann nicht statt. Recht als Antrieb der Entwicklung im Alleingang hat sich als ungeeignet erwiesen.

Die Beantwortung der zweiten Frage ist weit schwieriger. Die westlichen Geberländer tendieren dazu, die Charakteristika des westlichen Entwicklungsweges, nämlich ihr Verhältnis von Individuum und Staat, sowie ihre staatlichen Organisationsprinzipien universalistisch zu sehen und für eine unverzichtbare Entwicklungsvoraussetzung zu halten. ${ }^{112}$ Sie reflektieren hierbei die Zusammenhänge von wirtschaftlichen, politischen, administrativen, gesellschaftlichen und rechtlichen Strukturen ${ }^{113}$ auf ihrem jeweiligen Erfahrungs- und historisch geprägten Theoriehintergrund. Hinzu kommt, dass sie ihr spezifisches Menschenbild mit diesem Verständnis verschränken, teils weil sie es ebenfalls für entwicklungsnotwendig halten, teils als eigenständiges, sozusagen zivilisatorisches Bestreben mit missiona-

108 Falke, Fn 105, S. 57-61 zu den einzelnen Handlungsfeldern deutscher Rechtsstaatsförderung.

109 Meinecke, Fn 2, S. 120.

110 Meinecke, Fn 2, S. 62.

111 Max Weber, Gesammelte Aufsätze zur Religionssoziologie, Band 1, Herausgegeben von Johannes Winckelmann, Tübingen 1988, S. 252.

112 Vgl. die Nachweise bei Dutt, Fn 2, S. 42; s. auch Senghaas, 1982, Fn 82 passim.

113 Neumann, Fn 4, S. 223: so sieht die funktionelle Konzeption der Neuen Institutionenökonomie Recht als notwendige Wachstumsvoraussetzung. 
rischem Charakter. Entwicklung wird als einheitlicher wirtschaftlich-politischer und zivilisatorischer Prozess begriffen.

Diese Verschränkung erfolgt im politischen Bereich im Postulat demokratischer Herrschaft, im rechtlichen in den Prinzipien, die unter dem Begriff ,Rule of Law“ zusammengefasst als auf einander bezogene Handlungszusammenhänge gesehen werden und Frucht der westlichen historischen Entwicklung und ihrer geistesgeschichtlichen Reflexion sind. Als ihr Kernbereich werden verbreitet die Menschenrechte betrachtet, die auch als Basis des Verhältnisses von Individuum und Staat gesehen werden. ${ }^{114}$ Diese Sichtweise kommt etwa in der Sichtweise der Universalität der Menschenrechte zum Ausdruck, die deren Transplantation einerseits zur Pflichtaufgabe andererseits zu einem Problembereich und gelegentlich zum Stein des Anstoßes bei den Empfängern macht.

Daneben nimmt im Rahmen der Rule of Law- und Good Governance-Ansätze die Auseinandersetzung mit der Frage des Zusammenhangs von Rechtsstaat und Demokratie ${ }^{115}$ breiten Raum ein sowie die Frage, ob Demokratie Entwicklungsvoraussetzung sei ${ }^{116}$, während das bei Rule of Law nicht ernsthaft bestritten wird. Es wird gelegentlich darauf verwiesen, ${ }^{117}$ Rule of Law sei auch in nicht demokratischen Staaten ${ }^{118}$ möglich. Hierfür können historische Beispiele angeführt werden, etwa das Zweite Deutsche Kaiserreich, wie auch gegenwärtige, vor allem die Republik Singapur. Thematisiert werden auch die Interdependenzen von Demokratie, Marktregulierung und Rechtsstaatlichkeit, ${ }^{119}$ also ob wirtschaftliche oder politische Bedingungen den Primärimpuls zur Entwicklung darstellen. Ferner werden die Interdependenzen einzelner Rechtsstrukturen ins Kalkül gezogen.

Insgesamt ist die Meinung wohl herrschend, unter Rule of Law als notwendigem Entwicklungserfordernis seien nicht nur funktionsnotwendige Rechtsstrukturen $\mathrm{zu}$ verstehen, sondern vor allem auch ein spezifisch westliches Grundverständnis von Staatlichkeit, das bestimmte Freiheitssphären des Individuums gegenüber dem Staat, ${ }^{120}$ bestimmte staatliche Handlungsmaximen wie Fürsorgepflichten und Kontrollierbarkeit nebst Verantwortlichkeit des Staatsapparates gegenüber seinen Bürgern umfasst.

114 Etwa Sobota, Fn 101, S. 65 ff; Danilo Zolo, The Rule of Law. A Critical Reappraisal, in: Pietro Costa, Danilo Zolo (eds) with the Cooperation of Emilio Santoro, The Rule of Law, History, Theory and Criticism. Law and Philosophy Library, Vol. 80, Dordrecht 2007, S. 3 - 72, S. 31; vgl. auch Neumann, Fn 4, S. 213 ff mwN.

115 Zum Verhältnis Rechtsstaat-Demokratie, s. Neumann, Fn 4, S. 216; Zolo, Fn 114, S. 32.

116 Zolo, Fn 114, S. 32.

117 Zolo, Fn 114, S. 32.

118 Sieht man genauer hin, so gab es nennenswerte Rule of Law Elemente bisher nur in nicht voll aus demokratisierten Staaten die eine politische Struktur mit demokratischen Elementen aufwiesen. Charakteristisch sind dort identifizierbare von Rule of Law ausgegrenzte Bereiche, in der Regel Privilegien der Herrschenden.

119 Nelken, Fn 15, S. 41.

120 Neumann, Fn 4, S. 205. 
Wenn man sich vergegenwärtigt, dass es auch im absolutistischen Staat der europäischen Geschichte Regeln gab, die im Allgemeinen akzeptiert wurden und Legitimität entfalteten, wozu Privilegien Herrschender zählten, ${ }^{121}$ die mit dem heutigen Verständnis von Rule of Law unvereinbar wären, wird allerdings die Kontextualisierung von Recht, seine „kulturelle Logik“122 sichtbar.

Das heutige Verständnis von Rule of Law ist Teil des westlichen Menschenbildes ${ }^{123}$ und ein Produkt der Aufklärung. ${ }^{124} \mathrm{Ab}$ dem 16. Jahrhundert änderte sich in Europa das Verhältnis von Individuum, Gesellschaft und Staat zueinander. Das Mehrprodukt und damit auch staatliche und gesellschaftliche Organisationsfähigkeit erhöhten sich. Es begannen ökonomische und politische Verteilungskämpfe und es standen allen Akteuren, vor allem aber dem staatlichen Bereich vermehrte Ressourcen zur Verfügung, die durch immer besser organisierte Staatsapparate entweder zur vermehrten Schaffung öffentlicher Güter oder zur Ausbeutung, Unterdrückung und Selbstprivilegierung eingesetzt werden konnten. Menschenrechte als Abwehrrechte des Individuums gegen den Staat entwickelten sich im Wesentlichen im 18. Jahrhundert. Menschenrechte entstanden damals als konzeptionelle Reaktionen auf Unrechtserfahrungen ${ }^{125}$ durch als erdrückend empfundene staatliche Macht. Wie die europäische Geschichte mit ihren schnell und stark wechselnden Handlungsszenarien zeigt, können Unrechtserfahrungen unter unterschiedlichen Handlungsbedingungen sehr unterschiedlich sein und deshalb auch unterschiedliche Vorstellungen über Menschenrechte hervorbringen. Durch die prinzipielle Unbegrenztheit der Erfahrungen erklärt sich das heutige Verständnis von Menschenrechten als ein dynamisches und offenes Modell ${ }^{126}$ und als Ausfluss eines sich dynamisch wandelnden Menschenbildes. ${ }^{127}$

So ist selbst unter den Industriestaaten eine abschließende inhaltliche Bestimmung, die Aufstellung eines abschließenden universellen Katalogs von Menschenrechten, strittig. ${ }^{128}$ Es besteht etwa beim zentralsten Menschenrecht, dem Recht auf Leben, Uneinigkeit ob das Verbot der Todesstrafe davon umfasst werden soll oder nicht.

Trotz der bedeutenden Dynamik, die in den letzten Jahren den inhaltlichen Bereich von Menschenrechten erheblich erweitert hat und immer mehr zu erweitern scheint, gehen west-

122 Wiater, Fn 58, S. 64.

123 Zolo, Fn 114, S. 30, der Rule of Law ohne gedankliche Verbindung mit dem westlichen Menschenbild für "inconceivable" hält.

124 Zur Geschichte Costa, Fn 101, S. 77.

125 Christine Meyer, Menschenrechte in Afrika. Regionaler Menschenrechtsschutz als Herausforderung an menschenrechtliches Universalitätsdenken. Leipziger Schriften zum Völkerrecht, Europarecht und ausländischen öffentlichen Recht, Band 22, Baden-Baden 2012, S. 67.

126 Im 20. Jahrhundert wurden Menschenrechte um eine zweite Dimension erweitert: Teilhaberechte, allgemein als 2. Generation der Menschenrechte bezeichnet. Eine sogenannte 3. Generation entwickelte sich in den letzten Jahrzehnten als kollektive (Abwehr- und Teilhabe) Rechte.

127 Ausführlich zu den verschiedenen Ansätzen bzgl. Menschenrechten Meyer, Fn 125, S. 22 ff.

128 Zolo, Fn 114, S. 38. 
liche Länder jedoch in der Regel davon aus, Menschenrechte seien vorrechtlich, universell, ahistorisch und dem Menschen sozusagen angeboren. Sie determinierten daher die Rechtsordnung von außen und stünden deshalb nicht zur Disposition nationaler Gesetzgeber.

Diese Sichtweise hat jedoch auch Kritik hervorgerufen. Bereits 1946 findet sich in einer Stellungnahme zur UN-Charta ${ }^{129}$ der vor allem von Rechtsethnologen vertretene Standpunkt, Menschenrechte seien relativ und kulturbedingt, die Forderung ihrer Implementierung in Entwicklungsländern mit anderem kulturellen Hintergrund sei „kulturimperialistisch". ${ }^{130}$ Menschenrechte, wurde hervorgehoben, seien eine westliche Erfindung und dem kulturellen Kontext anderer Völker fremd und ungemäß.

In den letzten Jahren wurde gegen dieses westliche Verständnis von Rule of Law und Menschenrechten ein ambitionierter asiatischer Gegenentwurf in Theorie und vor allem Praxis eingeführt. Diese Sichtweise bestreitet weder die Wichtigkeit von Eigentumsrechten und anderen ökonomischen und sozialen Rechten, noch insbesondere die Notwendigkeit einer regelgeleiteten, kenntnisvollen und willkürfreien Staatstätigkeit für Entwicklung, konzipiert sie jedoch nicht als Rechenschaftspflicht des Staates und Forderungsrechte gegen diesen im Sinne von Menschenrechten. Sie hält politische Teilhaberechte und politische Kontrollfunktionen der Zivilgesellschaft für nicht entwicklungsnotwendig, ja entwicklungshemmend und die Staatstätigkeit behindernd. Diese solle grundsätzlich von der Zivilgesellschaft unkontrolliert erfolgen.

Geprägt werden hierfür Begriffe wie „Asian Values“, die als „konfuzianisch“ und durch die chinesische Geschichte geprägt apostrophiert werden, womit, nach den Worten von Lee Kuan Yew, dem früheren Premierminister von Singapur, gemeint ist, dass politische Rechte hintangestellt werden, bis wirtschaftliche Entwicklung ein bestimmtes Niveau erreicht hat. ${ }^{131}$ Weiter wird von Vertretern dieser Auffassung vorgebracht, zu „Asian Values“ gehöre eine Art Sozialkontrakt zwischen Gesellschaft und Regierung, ${ }^{132}$ der dieser autoritative Befugnisse einräume.

Legitimes Interesse ist nach dieser Auffassung im Wesentlichen nur das der Gemeinschaft. Das Interesse der Gemeinschaft wird in China traditionell durch eine Regierung vertreten, die von hohen ethischen Werten geleitet wird ${ }^{133}$ und die Ordnung und Stabilität garantiert, Werte, die eingebettet sind in die ostasiatischen Wertstrukturen, wo Harmonie an sehr hoher Stelle der Wertehierarchie steht. Die Legitimität des chinesischen, stark hierar-

129 The Executive Board, American Anthropological Association, 1946, S. 539-543. The Executive Board, American Anthropological Association, Statement on Human Rights, in: American Anthropologist, Vol. 49, No 4, (1946) Part 1, S. 539-543.

130 Wiater, Fn 58, S. 70.

131 Alice Ehr-Soon Tay, Asian Values and the Rule of Law, in: Pietro Costa, Danilo Zolo (eds), with the Cooperation of Emilio Santoro: The Rule of Law, History, Theory and Criticism. Law and Philosophy Library, Vol. 80, Dordrecht 2007, S. 565-586, S. 575.

132 Tay, Fn 131, S. 576.

133 Martin Jacques, When China Rules the World. The End of the Western World and the Birth of a New Global Order, 2nd edition, London 2009, S. 261. 
chisch strukturierten Staates ergibt sich aus der ihm zugeschriebenen Wächterfunktion für die chinesische Lebensform. Er wird quasi als Verkörperung der chinesischen Kultur gesehen. ${ }^{134}$ Die Garantie der Qualität staatlichen Handelns und vor allem der Schutz vor Willkür erfolgt in diesem System also durch Internalisierung von Werten und eine entsprechende Erwartungshaltung der Bevölkerung. Die Gesellschaft setzt ihrem Staat keine institutionellen Grenzen der Respektierung individueller Rechte. ${ }^{135}$

Bei näherem Hinsehen wird jedoch deutlich: An „Asian Values“ ist nichts spezifisch konfuzianisch, asiatisch oder kulturbedingt. Es geht vielmehr um ein Staatsverständnis, das in allen vorindustriellen Gesellschaften stets herrschend war $^{136}$. In allen vorindustriellen Gesellschaften steht die Schutzbedürftigkeit des Individuums und damit seine Integration in die Gemeinschaft im Vordergrund. Individuelle Freiheiten sind in einer alternativenarmen Umwelt mit niederer Produktivität dysfunktional für Gesellschaft wie Individuum. Alle traditionellen Staaten weisen diese Struktur auf und alle westlichen Industriestaaten durchliefen dieses Stadium in ihrer Geschichte. ${ }^{137}$

Es handelt sich bei „Asian Values" um die legitimatorische Verortung eines vorindustriellen Entwurfs des Verhältnisses von Bürger und Staat, vorgetragen mit der Ambition entwicklungsfördernder Äquivalenz. Um eine kulturlegitimierte Gegenposition zur westlichen aufzubauen, haben sich Herrschende ${ }^{138}$ auf eine als konfuzianisch bezeichnete Rechtfertigungslinie zurückgezogen, wobei sie allerdings deren Voraussetzungen nach ihren jeweiligen Bedürfnissen interpretieren. ${ }^{139} \mathrm{Ob}$ sich intern erfolgreiche Gegenvorstellungen hiergegen entwickeln, hängt wohl von der wirtschaftlichen Entwicklung und den mit ihr notwendig verbundenen gesteigerten Entscheidungsalternativen und Entscheidungszwängen für das Individuum wie den Staat ab. Ansätze sind in Korea, Taiwan und auch - unasiatisch konfrontativ - in Hongkong bereits zu sehen.

Entscheidend ist, dass China in seiner gesamten eindrucksvollen Geschichte niemals das Stadium eines industrialisierten Landes mit den, einem solchen vorgegebenen Handlungsbedingungen erreicht hat. Es steht jetzt erstmalig in seiner Geschichte an der Schwelle der Industrialisierung. Ob das chinesische System jedoch für ein industrialisiertes Land taugen wird, muss an dieser Stelle nicht diskutiert werden, da es sich bei den afrikanischen nicht um Industrieländer handelt sondern um Länder, die noch einen weiten Weg dorthin vor sich haben.

134 Jacques, Fn 131, S. 266.

135 William Theodore de Bary, Asian Values and Human Rights. A Confucian Communitarian Perspective, Cambridge 1998; Randall Peerenboom (ed), Asian Dimensions of Rule of Law in Twelve Asian Countries, France and the US, London 2004.

136 Dieter Senghaas, Zivilisierung wider Willen. Der Konflikt der Kulturen mit sich selbst, Frankfurt a. M. 1998, S. $178 \mathrm{ff}$.

137 Senghaas, Fn 136, S. 181.

138 Interessanterweise auch in Afrika (in Ruanda kommen bereits die Schulbücher aus Singapur).

139 Tomuschat, Fn 12, S. 76. 
Das chinesische System, das in Jahrtausenden durch trial and error und deren reflektorische Verarbeitung in einem großen Flächenland mit sehr großer Einwohnerzahl entstanden ist, ist für Afrika jedoch selbst bis zur Erreichung eines bescheidenen Industrialisierungsgrades untauglich, denn alle seine Voraussetzungen fehlen dort. Afrikanische Stämme mögen im Einzelfall Mechanismen von administrativer Qualität und auch Verantwortlichkeit ausgebildet haben oder auch nicht, eine Entwicklung zu institutionen- (nicht personen-) geprägten Flächenadministrationen und erheblich über Subsistenzniveau hinausgehenden Mehrprodukten (wenn auch in vorindustriellen Dimensionen) und dem Umgang mit ihnen, haben sie nicht durchgemacht und deshalb auch nicht reflektorisch verinnerlicht. Eine der chinesischen auch nur annähernd vergleichbare Traditionsethik, die von der Öffentlichkeit eingefordert würde, ist bei afrikanischen Staaten daher nicht auszumachen. Damit fehlt die entscheidende Stütze des chinesischen Systems.

Auf der anderen Seite geben die im Vergleich ungleich größeren Handlungsspielräume heutiger Staatsapparate durch gesteigertes volkswirtschaftliches Mehrprodukt (wozu insbesondere auch Transferleistungen nach Afrika gehören) und technische Möglichkeiten afrikanischen Staatsapparaten Machtpotenziale über zivilgesellschaftliche Gruppen und Individuen, wie sie Staaten früherer Zeiten, auch China, nicht annähernd zur Verfügung standen. Je größer diese Machtpotenziale sind, desto mehr kann damit in das Leben der Zivilgesellschaft und von Individuen eingegriffen werden und desto mehr entsteht ein Bedürfnis nach Kontrolle und Verantwortlichkeit von Staatsapparaten. Da Gemeinwohlorientierung und Selbstdisziplin aus den genannten Gründen im afrikanischen kulturellen Kontext keine Optionen darstellen, die Funktionsfähigkeit eines Staates und den Schutz seiner Bevölkerung vor gewaltsamen Willkürakten zu sichern, bleibt deshalb für afrikanische Staaten nur die auf Misstrauen beruhende Rule of Law, um eine geordnete Staatstätigkeit zu erreichen.

Der spezifische Appeal von „Asian Values“ für afrikanische Regierungen liegt darin, dass hier ein wirtschaftlicher Entwicklungsweg aufgezeigt wird ohne die westlichen Werte von Verantwortlichkeit von Staatsapparaten und demokratischer Kontrolle. Die Vorbildfunktion ist deshalb so stark, weil es afrikanischen Machthabern gerade darauf ankommt, unkontrolliert zu agieren und in Gesellschaften Macht und Reichtum anzuhäufen, in denen diese nicht nur praktisch die einzigen sozialen Positionsmarker darstellen, sondern auch eine Art „Lebensversicherung“"140 in weitgehend anomischen und instabilen Umwelten. Und in der Entwicklung von Singapur und China sehen sich diejenigen bestätigt, die die Ansicht vertreten, wirtschaftliche Dynamik könne von sozialer und politischer entkoppelt werden. ${ }^{141}$

Eine stabile und wägbare Alternative zu den Prinzipien von Rule of Law und Demokratie scheint für Afrika nicht ersichtlich. Hierbei können freilich Einzelheiten, insbesondere

140 Mit durchaus auch kollektiven Bezügen: Machthaber sichern ihrem eigenen Stamm/Clan eine vorteilhafte Ausgangsposition für die, oft auch gewaltsame, Positionierung in der nationalen Arena nach ihrem Ausscheiden aus dem Amt.

141 S.o.Fn 90. 
individuelle Freiheiten, unterschiedlich ausgeprägt sein, ${ }^{142}$ müssen nicht unbedingt europäischen Mustern folgen, sondern entwickeln tatsächlich meist eine eigene Dynamik.

Die legitimatorische Basis für die rechtliche Strukturierung des Staates, auch des Entwicklungsstaates, ${ }^{143}$ ist letztlich die Bezogenheit des Staates auf die in seinen Grenzen lebenden Individuen und seine Aufgabe, öffentliche Güter für dieselben zu produzieren, die je nach den historischen Bedingungen variieren, insbesondere auch in der Belastung, der Individuen dabei ausgesetzt sein müssen und dürfen.

Hieraus leitet sich das Verständnis von Menschenrechten als gleichzeitig ahistorisch wie auch dynamisch her. Es liegt in der Beziehung der Konstitution des Menschen mit der Struktur seiner Umwelt, auf bestimmte externe Anforderungen sinnvoll nur mit bestimmten kollektiven Verhaltensmustern reagieren zu können, um als Gruppe und Individuum in der Gruppe zu überleben. Diese Verhaltensmuster, zu Regeln institutionalisiert, lassen sich bei Parallelitäten der Lebensverhältnisse als Konstanten und damit als externe „natürliche“ Grundlagen rechtlicher Gestaltung ${ }^{144}$ begreifen, hängen allerdings in Randbereichen auch von individuell und kollektiv veränderlichen Perzeptionen ab. ${ }^{145}$ Sie besitzen, auch durch die kulturellen Konditionierungen der Konzeptualisierenden, eine gewisse Variationsbreite, die insbesondere eine Variationsbreite des metatheoretischen Verständnisses was „natürlich" ist, ${ }^{146}$ einschließt.

In Afrika wird deutlich sichtbar, wie Umweltbedingungen und die Möglichkeiten der Reaktionen auf diese den Grad der Einbindung des Individuums in soziale Handlungszusammenhänge determinieren. ${ }^{147}$ Freiräume gegenüber der und Forderungen an die Gemeinschaft sind immer nur soweit möglich, als deren materielle Mittel und Funktionsfähigkeit nicht gefährdet werden.

142 Dieter Grimm, Stufen der Rechtsstaatlichkeit: Zur Exportfähigkeit einer westlichen Errungenschaft, In: Juristenzeitung 64 (2009) S. 596-600.

143 Hierzu Wiater, Fn 58, S. 44.

144 Ihre humankonstitutionelle Komponente, dass Menschen nur in bestimmter Weise sinnvoll auf Anforderungen der Umwelt reagieren können, lässt sich als ahistorisch und vorrechtlich, nicht zur Verfügung der Beteiligten stehend und die Recht- und auch Sozialordnung von außen determinierend verstehen. Hier zeigt sich die Überlappung der Kontroverse Absolutheit-Kulturrelativismus mit einer andern: Naturrecht-Rechtspositivismus. Nach dem hier dargelegten Verständnis können Menschenrechte nicht rechtspositivistisch als willkürlich gesetzt verstanden werden, sondern werden in ihren Umrissen durch die conditio humana festgelegt und erfahren Veränderungen durch die Veränderungen der Handlungsbedingungen und deren jeweilige kulturbedingte Reflexion.

145 Meyer, Fn 125, S. 34: Interaktion zwischen Kultur und Umwelt des "lernfähigen Menschen".

146 Wiater, Fn 58, S. 125.

147 Benezet Bujo, Afrikanische Anfrage an das europäische Menschenrechtsdenken, in: Johannes Hoffmann (Hrsg.), Begründung von Menschenrechten aus der Sicht unterschiedlicher Kulturen, Frankfurt a. M. 1991, S. 211-225, S. 216. 
So kann man sagen, dass es in allen menschlichen Gesellschaften die Notwendigkeit staatlicher Macht und ihrer Begrenzung gibt, ${ }^{148}$ beide aber in ihrem jeweiligen Umfang von den historischen Bedingungen im Einzelnen abhängen. Das Verhältnis von Staat und Individuum muss daher in jeweils adäquaten, in den sich ebenfalls unter den historischen Bedingungen bildenden Legitimationsstrukturen verorteten, Rechtskonstruktionen gefasst werden. Diesen Rechtskonstruktionen gemeinsam sind Kriterien der Rücksichtnahme der Gemeinschaft auf das Individuum. Menschenrechte sind hierbei die Version der westlichen Gesellschaften.

In afrikanischen Gesellschaften gibt es traditionell andere Schutzkonzepte und Schutzmechanismen für das Individuum. ${ }^{149}$ In afrikanischen Gesellschaften (sowie asiatischen und allen islamischen) stößt bereits die westliche, manchmal geradezu antagonistische Gegenüberstellung von Individuum und Gruppe, ${ }^{150}$ also auch Staat, auf Unverständnis, wird doch das Individuum nur durch die Vermittlung der Gruppe als existenz- und handlungsfähig gesehen. Rechte des Individuums gegenüber der Gruppe werden nicht als auf Freiheit, sondern als auf Schutz gerichtete konzipiert. ${ }^{151}$ Neue materiell-rechtsstaatliche Arrangements müssen daher in Afrika im Einzelnen auf Kompatibilität geprüft werden und können auch nicht abrupt erfolgen.

Der in internationalen Abkommen festgehaltene Kanon der Menschenrechte stellt letztlich eine „Schnittmenge“ ${ }^{\text {"152 }}$ dar von Überzeugungen ${ }^{153}$ über gemeinsame Standards, ${ }^{154}$ von „cross-cultural universals“. ${ }^{155}$ Problematisch ist hierbei sowohl, dass Geber nicht wissen, ob afrikanische Zustimmung in internationalen Abkommen auf Überzeugung oder politischem Kalkül beruht (und im zweiten Fall in der Praxis unterlaufen wird) als auch, dass die entsprechenden Vorstellungen in Afrika stark fraktioniert sind.

148 Meyer, Fn 125, S. 32 spricht von "Core rights" (innerhalb eines "deskriptiv-dynamischen" Ansatzes).

149 Meyer, Fn 125, S. 38, 40.

150 Hierzu Alliot, Fn 18, S. 194.

151 Bujo, Fn 147, S. 216.

152 Wiater, Fn 58, S. 46.

153 Es handelt sich letztlich um Werte, die als solche verallgemeinerungsfähig (Juergen Habermas, Faktizität und Geltung. Beiträge zur Diskurstheorie des Rechts und des demokratischen Rechtsstaats, Frankfurt a. M. 1998) sind. Hierzu gehören, wohl als historische Konstante, auch das Streben nach Steigerung von Lebensqualität durch entsprechende Produktion öffentlicher Güter, etwa Hebung von Wohlstand und Gesundheit (Christian Roschmann, Menschenrechte und Armut in Afrika, in: Recht von innen: Rechtspluralismus in Afrika und anderswo. Justice from within: Legal Pluralism in Africa and beyond. Festschrift Manfred O. Hinz anlässlich seines 75. Geburtstags. Liber amicorum Manfred O. Hinz in celebration of his 75th birthday, herausgegeben von/ edited by Oliver C. Ruppel and Gerd Winter, Hamburg 2011, S. 287 - 314, S. 429), das im Verständnis westlicher Gesellschaften zu Teilhaberechten führte, wie den Menschenrechten der 2. Generation.

154 Tay, Fn 131, S. 584; Alison Dundes Rinteln, International Human Rights. Universalism versus Realism, Newbury Park Ca 1990, S. 48.

155 Dundes Rinteln, Fn 154, S. 48. 


\section{Entwicklungsbedingungen als Ursachen und Wirkungen}

Recht im Verhältnis von Bürger und Staat und damit Rule of Law, durchdringt jedoch fast alle Handlungen des Staates im Verhältnis zum Individuum. Besonders deutlich wird das bei der Frage der unabhängigen Kontrolle staatlicher Einzelhandlungen, ihrer Justiziabilität.

Bei der Vermeidung solcher Kontrolle verstärken sich zwei heterogene Erscheinungen als Hindernisse gegenseitig, nämlich afrikanische Legitimitätsvorstellungen und Interessen politischer Eliten. Für die Masse der Bevölkerung handelt es sich beim Handeln des modernen afrikanischen Staates um eine fremde Erscheinungsform, oft zu fremd um sie durch systeminterne Kontrollen auch nur „bändigen“ zu wollen.

In Entwicklungsländern ist sowohl das gestiegene Mehrprodukt als auch die Organisationsfähigkeit von Staatsapparaten in sehr hohem Maße auf Geberleistungen zurückzuführen. Staatsapparate verfügen über Ressourcen und Macht, deren Voraussetzungen sie und die nationalen Gesellschaften für die sie agieren oder zu agieren vorgeben in erheblichem Maße nicht selbst produziert haben. Es scheint, dass diese Tatsache sowohl bei den Eliten wie bei den relevanten Öffentlichkeiten einen erheblichen legitimatorischen Einfluss auf den Umgang mit Ressourcen hat, gerade auch im Verhältnis zum Bürger. Je mehr Ressourcen dem Staatsapparat jedoch zur Verfügung stehen, desto mehr kann entweder für Wirtschaftsförderung und ein soziales Netz, oder für einen Unterdrückungsapparat und persönliche Bereicherung von Politikern aufgewendet werden.

Die beschriebenen Machtstrukturen und ihre kulturelle Verankerung führen vielfach da$\mathrm{zu}$, dass Rechtsregeln nicht beachtet werden. Impunität für Inhaber von staatlichen Machtpositionen, die diese missbrauchen, gibt es seit es Staaten gibt. Mangel an Rechtsstaatlichkeit äußert sich nicht in erster Linie darin, diese oder jene Regeln zu missachten, sondern in der mangelnden Bereitschaft, staatliche Regeln überhaupt einzuhalten.

Dass der Bruch von Regeln mit Machtfülle korreliert, wird von Bevölkerungen, die diesem Phänomen seit Jahrtausenden ausgesetzt sind, als notwendiges Übel angesehen. Impunität im Kontext sich entwickelnder Staaten bewirkt jedoch, dass die für eine wirtschaftliche, politische und gesellschaftliche Entwicklung erforderliche Rechtssicherheit fehlt, ungeachtet dessen ob Gesetze auf dem Papier ,gelten“, oder nicht. Impunität jedoch ist unter Staatsbediensteten aller Ränge fast überall in Afrika ein Massenphänomen. Es dürfte in der menschlichen Natur liegen, sich - tendenziell durchaus auch illegal - zu bereichern, wenn dies gefahrlos möglich ist.

Politische und bürokratische Innenkontrolle eines Staatsapparates wird es nur geben, wenn es Außenkontrolle gibt. Diese aber gibt es nur durch eine kontrollfähige Zivilgesellschaft, deren Bedeutung wiederum eng von den wirtschaftlichen und politischen Strukturen abhängt. Blickt man auf die nachholende Entwicklung mancher Industriestaaten, etwa von Japan oder Skandinavien, so wird erkennbar, dass beide Faktoren nur einigermaßen gleichzeitig, allmählich und vor allem verbunden wachsen.

Nur eine machtvolle, organisationsfähige Zivilgesellschaft kann bestimmenden Einfluss auf Legislative und Exekutive ausüben und bestehende Strukturen delegitimieren, voraus- 
gesetzt sie entwickelt parallel eine entsprechende rechtsstaatlich-demokratische Sensibilität. Machtvoll kann eine Zivilgesellschaft jedoch nur werden, wenn sie entsprechend produktiv ist, ein Mehrprodukt zu erwirtschaften, das ihre Einkommensbasis signifikant vergrößert und das mindestens nicht wesentlich hinter den bürokratischen Renten des Staatsapparates zurückbleibt, die, nicht zuletzt durch Transferleistungen der Geber, in der einen oder andern Form ,erwirtschaftet“ werden. Hierzu sind funktionierende Massenmärkte notwendig, die wiederum nur ohne Interferenzen machtvoller Bürokraten entstehen können. Damit schließt sich der Kreis und es wird erkennbar: Entwicklungshindernisse bedingen sich gegenseitig. Rule of Law ist einerseits Voraussetzung, andererseits Folge anderer Entwicklungsvoraussetzungen. Rule of Law ist daher nicht isoliert transferierbar.

Vergrößerung der Einkommensbasis und damit der wirtschaftlichen und politischen Macht der Mittelklassen durch Investitionen zur Schaffung von Massenmärkten, Institutionalisierung von Rechtsregeln, Ethos der Befolgung solcher und der Verantwortlichkeit, Organisation einer kritischen Zivilgesellschaft und Bildung entsprechender Legitimitätsvorstellungen und auch Pfadabhängigkeiten und Kenntnisse müssen „ko-evolutiv “156 entstehen und mehr oder weniger gleichzeitig wachsen, wie dies auch in der europäischen geschichtlichen Dynamik so war, und sie müssen auch so von Gebern gefördert werden.

Aus Gründen der Ressourcenknappheit muss dabei im Wesentlichen auf Strukturen, die sich im Weg von trial and error in entwickelteren Staaten gebildet haben, zurückgegriffen werden und diese, ebenfalls im Weg von trial and error, angepasst werden. Es wäre zu kostenträchtig und langwierig, stets neue aufwändige Konstruktionen zu erdenken, um den jeweiligen Gegebenheiten gerecht zu werden. ${ }^{157}$

Diese Zusammenhänge haben allerdings auch demokratiefeindliche autokratische Regime erkannt. Sie reagieren in den letzten Jahren mit dem Aufbau immer restriktiverer rechtlicher Hindernisse für die ausländische Unterstützung zivilgesellschaftlicher Organisationen mit der Begründung, es handele sich bei solcher um eine Einmischung in die Innenpolitik $^{158}$ und Verletzung ihrer Souveränität. An dieser Stelle wird die scharfe Konfliktlinie sichtbar zwischen demokratie- und rechtsstaatsfeindlichen Staatsapparaten, deren Ziel Machterhaltung ist, und den Entwicklungsvorstellungen der Geber.

In allen Fällen nachholender Entwicklung war eine entwicklungsorientierte und vor allem verantwortungsbewusste Regierung und Verwaltung im Spiel - im historischen Vergleich eine Ausnahme ${ }^{159}$-, die in Afrika jedoch, jedenfalls gegenwärtig, erkennbar in den

156 Teubner, Fn 43, bezeichnet diese Gesetzmäßigkeit als „Koevolution“ (im Titel seines Beitrags). Habermas, Fn 153, S. 138, benutzt den Begriff „gleichursprünglich“ (allerdings für das Verhältnis von Recht und Legitimitätsvorstellungen) zur Darstellung des Koppelungsverhältnisses zweier Handlungszusammenhänge.

157 Meinecke, Fn 2, S. 117.

158 Beispielhaft sei die sehr restriktive äthiopische Charities and Securities Proclamation 621/2009 genannt oder die in mehreren Ländern beobachtete Praxis, ausländische Institutionen des Landes zu verweisen und teilweise deren Personal mit Prozessen zu überziehen.

159 Senghaas 1982, Fn 82; Roschmann 1999, Fn 85. 
meisten Ländern nicht besteht. Nachdem Geber in Entwicklungsländern einen tatsächlichen Einfluss über Transferleistungen ausüben, könnten sie jedoch auf die geordnete simultane Entwicklung solcher Strukturen Einfluss ausüben, allerdings, zur Erreichung einer kritischen Masse, nur koordiniert.

\section{Rechtstransfer}

Für Rechtstransfer heißt das, es ist nicht zielführend, eine gesteigerte Transfertiefe bei der Regelung juristischer Einzelheiten zu entwickeln, deren Früchte in den andern genannten Handlungszusammenhängen - oder auch nur in anderen juristischen Teilbereichen - noch nicht verarbeitet werden können, es sei denn es handelt sich um technische Details wie etwa elektronische Informationssysteme für Gerichte. So macht es etwa keinen Sinn, Rechte von Gewerkschaften im Einzelnen zu normieren, wenn solche noch nicht organisiert werden können, weil deren Organisationsfähigkeit von Machthabern noch gezielt unterlaufen werden kann. Außerdem können rechtliche Veränderungen nur im Einklang mit Veränderungen der Legitimitätsstruktur, in der sie verortet werden müssen, in einem „soft change“ erfolgen, das heißt allmählich. Es spricht daher manches dafür, Rechtsinnovationen in progressiver Anwendung zu allmählicher Änderung zu implementieren ${ }^{160}$ und nach und nach einzelne Elemente neuen Rechts zu übernehmen.

Alliot weist darauf hin, die Einführung von Recht europäischen Zuschnitts könne politisch von Staatsapparaten dosiert zu gezielten Verhaltensänderungen und damit als Mittel der Entwicklung eingesetzt werden. ${ }^{161}$ So könnten über tatsächliche Verhaltensänderungen gezielt Einstellungs- und Legitimitätsänderungen erreicht werden. Die Rechtsstrukturen würden sozusagen nicht als das ,hard law" als das sie in den Industrieländern konzipiert würden implementiert, sondern als ,soft law“, als Orientierung mit progressiver Anwendung, ${ }^{162}$ deren Einhaltung entweder nicht strikt erzwungen, oder selektiv nach Rastern von Erheblichkeit und Grob- Feinorientierung eingefordert werden könne. Mit der Zeit könne dann die Einhaltung immer restriktiver durchgesetzt werden, so dass ein allmählicher Gewöhnungsprozess eintrete.

Dieser Prozess kann von Gebern sachkundig unterstützt werden. Das gilt insbesondere für die genannten Rule of Law Elemente, wie Menschenrechte. Hinz ${ }^{163}$ schlägt vier Stufen der langsamen Einführung von Menschenrechten in afrikanischen Staaten vor: Ein großer Zeitrahmen der Einführung von Regelungen, ein noch größerer Zeitrahmen für die sanktionierte Verbindlichmachung, die Koppelung mit Pflichten und die Bezugnahme auf intrakul-

160 Fikentscher, Fn 28, S. 217 ff.

161 Alliot, Fn 18, S. 216.

162 Alliot, Fn 18, S. 217.

163 Manfred O. Hinz, Human Rights between Universalism and Cultural Relativism? The Need for Anthropological Jurisprudence in the Globalising World, in: Anton Boesl, Joseph Diescho (eds): Human Rights in Africa, Legal Perspectives on their Protection and Promotion, Windhoek 2009, S. 3-32, S. 20-26. 
turelle Anknüpfungspunkte. ${ }^{164}$ So kann etwa Meinungsfreiheit, die zweifellos für eine demokratische Machtkontrolle unerlässlich ist, dann, wenn sie kulturellen Regeln grob widerspricht, etwa in manchen Kulturen dann, wenn Autoritätspersonen kritisiert werden, nach und nach und mit inhaltlich variablen Akzenten institutionalisiert werden.

Es kann als Erfahrungstatsache gelten, dass die Änderung beachteten positiven Rechts, auch in Industrienationen, langfristige Einstellungs- und Verhaltensänderungen durch Bildung und Einübung von Pfadabhängigkeiten bewirkt.

Dem widerspricht nicht die am Scheitern der Law and Development-Bewegung beobachtete Tatsache, dass Recht nicht Entwicklungslokomotive sein kann. Es geht hier vielmehr um mehr oder weniger synchrone Entwicklung in Handlungszusammenhängen verbundener Strukturbereiche innerhalb von Staaten, wobei der rechtliche in vielen Fällen eine vorsichtig dosierte, beschränkte innovative Leitfunktion übernehmen kann. ${ }^{165}$ Der jeweils nächste Schritt wird dann unternommen, wenn die relevanten anderen Strukturbereiche dem ersten gefolgt sind. Wenn man die Geschichte Deutschlands im 19. Jahrhundert betrachtet, so stellt diese sich als ein zähes Ringen mit Gewinnen und Rückschlägen dar, zwischen demokratiefeindlichen, wettbewerbs- und verantwortlichkeitsaversen, rentenmaximierenden Eliten und emergenten demokratie- und rechtsstaatsorientierten marktaffinen Mittelklassen. Auch hier entwickelten sich Legitimitätsvorstellungen und Marktmacht in dieser Weise, sich sozusagen gegenseitig aufschaukelnd, wobei jedoch eine gewisse Leitdynamik wirtschaftlicher Entwicklung beobachtbar ist, sobald sie einmal in Gang gekommen war, wie dies auch in Ländern wie Nigeria oder Kenia, ganz zu schweigen von Indien oder China, sichtbar ist.

Was nicht übersehen werden kann, ist die Langfristigkeit dieser Dynamik, die auch am Beispiel Deutschlands erkennbar ist, das von 1819 bis 1949 um Rechtsstaatlichkeit gerungen hat, speziell um Menschenrechte noch länger. Entwicklungszusammenarbeit heißt dann aber auch langfristige, nachhaltige Stärkung der wirtschaftlichen Strukturen durch Entwicklung von Märkten und der Mittelklasse (und nicht der Staatsklasse), und Rechtstransfer in diesem Zusammenhang heißt vor allem auch, Stärkung von Legitimitätsvorstellungen durch Hilfe zur vorsichtigen und schrittweisen Erfüllung zunächst fremder Normen mit Leben. Das kann nur mit allmählich wachsender Intensität geschehen. Diese Entwicklung wird etwa deutlich bei der bereits feststellbaren veränderten Beurteilung der Frage, wer in Afrika welche Regeln brechen darf, die in neuester Zeit in Gerichtsverfahren gegen hochrangige afrikanische Politiker, vor allem auch vor dem Internationalen Strafgerichtshof, ${ }^{166}$ ihren Ausdruck findet.

Eine solche neue Strategie des Rechtstransfers heißt jedoch auch Abschied nehmen von manchen bisherigen Vorstellungen. Zielführend erscheint weder die Vermittlung von De-

164 Vgl. auch Roschmann 2011, Fn 153, S. 227, 228.

165 Alliot, Fn 18, S. 216: er , sucht die für die Entwicklung nötige Revolution zu vollenden”.

166 Dessen Aktivitäten in der Bevölkerung zwar sehr kontrovers beurteilt werden, jedoch damit bereits eine Legitimitätsreflexion und -debatte eingeleitet haben. 
tailwissen an Führungskräfte, wenn diesen Kenntnisse nahegebracht werden, deren Anwendung gerade zu vermeiden Teil ihres Rollenverständnisses ist, wie effektive Kontrollen des Verwaltungshandelns, weil sie ihre Machtbasis schmälern, noch erscheint zielführend, nicht verantwortungsgebundenen Regierungen Finanzmittel zu juristischen Innovationen zur Verfügung zu stellen; die Möglichkeiten über deren Verwendung zu täuschen sind zu mannigfach. Auch sollte in diesem Zusammenhang die Praxis, höhere Funktionsträger zu kostspieligen Besuchs- und Informationsreisen ohne nachhaltige Folgen einzuladen, überdacht werden.

Eine Entlastung der Rechtstransferbemühungen und auch eine Effektivitätssteigerung im Sinne einer Bündelungswirkung für den Rechtstransfer stellt die Ausgrenzung bestimmter nicht reformbedürftiger Rechtsgebiete im Wege der Institutionalisierung von rechtspluralistischen Lösungen dar.

Besonders in Ländern mit ethnisch diversifizierten Gruppen wird es den Bedürfnissen der einzelnen Bevölkerungsteile gerecht, jeder Gruppe, soweit dies möglich ist, ihr eigenes Recht zu belassen und staatlich insoweit nur noch Konfliktnormen anzuwenden. Dies wird etwa im Familien-und Erbrecht bereits in vielen Ländern ${ }^{167}$ erfolgreich praktiziert, nicht nur in Entwicklungsländern, sondern etwa auch in Deutschland. ${ }^{168}$

Rechtspluralismus ist sicher das sanfteste Mittel der Rechtsentwicklung, auch in Afrika. Man differenziert nach Rechtsgebieten. Diejenigen, bei denen das zu Entwicklung für notwendig gehalten wird, werden von staatlichen Instanzen entsprechend geformt. Diejenigen, bei denen das nicht so ist, werden belassen. Interessant ist dabei die Möglichkeit, nach verschiedenen Gesichtspunkten zu differenzieren. Zum einen ethnisch bzw. lokal. Jede Ethnie und jedes Gebiet kann ihr eigenes Recht behalten. Zum andern nach sozialen Gruppen auf einer Skala zwischen „keinerlei Adaption an westliche Regulierungsmechanismen“ und „hundertprozentiger Adaption“, etwa städtischer Eliten. So gibt es in verschiedenen afrikanischen Ländern die Möglichkeit, zwischen Eheregimen zu wählen, wobei das westliche und das traditionelle die beiden Enden eines Kontinuums darstellen können. Interessant ist auch die Differenzierung nach staatlichen Organisationsebenen, lokal, national und regional. ${ }^{169}$

Die Frage stellt sich dann allerdings, wieweit Rechtspluralismus ${ }^{170}$ gehen kann, eine Frage der eine gewisse Dynamik innewohnt, die unter Bedingungen der Migration auch In-

167 Etwa die kenianische Verfassung von 2010 anerkennt ausdrücklich eine pluralistische Rechtsordnung auf diesen Gebieten.

168 Für Deutschland: Art. 234, 235 EGBGB.

169 Hierzu instruktiv Katharina Ruppel-Schlichting/Oliver C. Ruppel, Applicable law and jurisdiction on the local, national and regional level: Facets of Legal and Judicial Pluralism in Namibia, in: Recht von Innen: Rechtspluralismus in Afrika und anderswo. Justice from within: Legal pluralism in Africa and beyond. Festschrift Manfred O. Hinz anlässlich seines 75. Geburtstages. Liber amicorum Manfred O. Hinz in celebration of his 75 th birthday, herausgegeben von/edited by Oliver C. Ruppel and Gerd Winter, Hamburg 2011, S. 287-314.

170 Zu Rechtspluralismus in Entwicklungsländern allgemein etwa Meinecke, Fn 2, S. 134-150 mwN. 
dustrieländer umfasst und dort eine parallele Diskussion hervorruft, ${ }^{171}$ die vielleicht in manchen Bezügen für Afrika fruchtbar gemacht werden kann.

Sicher kann Staats- und Verwaltungsrecht nur einheitlich in Verfassungen und Verwaltungsgesetzen, sowohl was Aufbau- wie Ablauforganisation betrifft, gesetzt werden. Hier sind in Einzelfällen Aussparungen für traditionelle Verwaltungen möglich, wie das etwa die Administration des Kabaka von Buganda in Uganda zeigt und für die es auch parallele Beispiele in Entwicklungsländern anderer Kontinente gibt. ${ }^{172}$ Auch diese Verwaltungen müssen jedoch auf vereinheitlichtes staatliches Verfahrensrecht zurückgreifen können soweit rechtsstaatliche Prinzipien tangiert sind, wie etwa das Recht auf rechtliches Gehör, die im Konfliktfall Vorrang haben müssen und wohl überall auch haben.

Die Grenzen möglichen Rechtspluralismus liegen bei der Nicht-Fragmentierbarkeit landesweiter Interaktionsnetze, etwa im Wirtschaftsrecht, im Verwaltungs- und Verfassungsrecht, also gerade den Rechtsgebieten, auf die es bei Entwicklung besonders ankommt. Außerdem ist der ordre public ${ }^{173}$ eines Landes auf allen Rechtsgebieten zu beachten. Es können keine grundsätzlichen Legitimitäts- und Wertvorstellungen und breit akzeptierte Handlungspfade verletzt werden. Diese Tatsachen machen Rechtspluralismus jedoch zu einem im Konfliktfall weichenden Prinzip.

Für direkten Rechtstransfer, das heißt für eine im Einzelfall nahezu hundertprozentige Regelungstiefe fruchtbar gemacht werden können die Handlungsfelder, die mehr oder weniger erst mit dem Modernisierungsschub entstanden sind oder problematisch wurden. Es sind vor allem die folgenden:

- „Wertfreies Regelverhalten“. ${ }^{174}$ Etwa die Regelung, ob man auf der rechten oder der linken Straßenseite fährt, ist ohne weiteres transferierbar. Sie bringt allen nur Nutzen.

- Eigentumsrechte an Land: Es existieren vielerorts Formen gemeinsamen oder semigemeinsamen Eigentums neben Individualeigentum. Hier scheint eine geografische Differenzierung nach Gebieten oder Gruppen möglich. Voraussetzung ist in jedem Fall ein funktionierendes Kataster- und Grundbuchwesen sowie in überbauten Gebieten eine Bauleitplanung und ein Nachbarschaftsrecht, so dass weder neben einem Luxushotel eine Schweinemästerei betrieben werden darf, noch eine Diskothek neben einem Kinderheim geduldet werden muss.

- Vertragsrecht. Auch in traditionellen Gesellschaften sind Verträge in aller Regel bindend. Deshalb stößt eine Entwicklung und Differenzierung dieses Rechtsgebiets wahrscheinlich auf Akzeptanz. In Grenzen sind daneben auch hier rechtspluralistische Regime im Sinne einer Rechtsformenwahl möglich.

171 Werner Menski, Fragwürdige Inklusion, Inakzeptable Exklusion, in: (E+Z) Entwicklung und Zusammenarbeit (11) 2009, S. 418-420.

172 Wie etwa die Verwaltung des Raja von Jogjakarta in Indonesien.

173 In Deutschland Art. 6 EGBGB: „Wesentliche Grundsätze des deutschen Rechts”. Diese gründen nicht unerheblich in kulturell geformten Werten und Vorstellungen.

174 Fikentscher, Franke, Köhler, Fn 27, S. 28. 
- Wirtschaftsrecht im weiteren Sinne, ${ }^{175}$ also etwa Gesellschaftsrecht, Insolvenzrecht, Urheberrecht und verwandte Rechtsgebiete. Hier haben wir es mit neuen Rechtsgebieten zu tun, die nicht der afrikanischen Lebenswelt entstammen und deshalb auch nicht mit Pfadabhängigkeiten, Legitimitätsvorstellungen, ja weitgehend überhaupt nicht mit Vorstellungen besetzt sind. Wer sich auf das hier geregelte Handlungsfeld begibt, sind die neuen Eliten und die sich formierende bürgerliche Mittelklasse, die eine in Industrieländern bewährte Regelung wohl hilfreich finden, während traditionelle Stammesstrukturen hiervon kaum betroffen sind. Deshalb wird es hier wohl wenige Widerstände gegen Rechtseinheit und Innovation geben.

Problematisch sind allerdings die Handlungsfelder, in denen die neuen Rechtsgebiete in bestehende Strukturen hineingreifen, wie vor allem im Arbeitsrecht und Arbeitsschutzrecht einschließlich des Rechts der sozialen Sicherungen.

\section{Schlussfolgerungen}

Es geht bei Rechtstransfer nach Afrika darum, zweigleisig zu fahren. Auf einigen Rechtsgebieten, die erst durch wirtschaftliche Entwicklung Relevanz erlangten und wo dem keine machtstrukturellen Hindernisse entgegenstehen, können Rechtsstrukturen in tendenziell voller Detailtiefe transplantiert werden und sollten dies auch.

Auf den andern Gebieten, insbesondere im Verhältnis des Bürgers zum Staat, müssen machtstrukturelle, kulturelle und instrumentelle Veränderungen mit wirtschaftlichen und rechtlichen Hand in Hand gehen und sie müssen auf einander abgestimmt sein. Fremde Rechtsstrukturen können nur allmählich eingeführt werden. Rechtliche Regelungen dürfen in ihrer Spezifik weder ihren eigenen Produktionsbedingungen vorauseilen noch unter sich eine dysfunktional heterogene Detailtiefe aufweisen. Die Einzelheiten können allerdings nur im Einzelfall ausgelotet werden. Hilfreich ist sicherlich die Intensivierung des begleitenden akademischen Diskurses.

175 Hierzu Dutt, Fn 2, S. 44. 